\title{
Using Non-Destructive Testing to Predict Bending Modulus of Carbon Infiltrated-Carbon Nanotubes
}

Fred Stile Fagergren

Brigham Young University

Follow this and additional works at: https://scholarsarchive.byu.edu/etd

Part of the Mechanical Engineering Commons

\section{BYU ScholarsArchive Citation}

Fagergren, Fred Stile, "Using Non-Destructive Testing to Predict Bending Modulus of Carbon InfiltratedCarbon Nanotubes" (2018). Theses and Dissertations. 8817.

https://scholarsarchive.byu.edu/etd/8817

This Thesis is brought to you for free and open access by BYU ScholarsArchive. It has been accepted for inclusion in Theses and Dissertations by an authorized administrator of BYU ScholarsArchive. For more information, please contact scholarsarchive@byu.edu, ellen_amatangelo@byu.edu. 


\title{
Using Non-Destructive Testing to Predict Bending Modulus of Carbon Infiltrated-Carbon Nanotubes
}

\author{
Fred Stile Fagergren
}

\begin{abstract}
A thesis submitted to the faculty of Brigham Young University in partial fulfillment of the requirements for the degree of

Master of Science
\end{abstract}

\author{
Brian D. Jensen, Chair \\ Richard Vanfleet \\ Eric Homer
}

\section{Department of Mechanical Engineering \\ Brigham Young University}

Copyright (C) 2018 Fred Stile Fagergren

All Rights Reserved 


\section{ABSTRACT \\ Using Non-Destructive Testing to Predict Bending Modulus of Carbon Infiltrated-Carbon Nanotubes \\ Fred Stile Fagergren \\ Department of Mechanical Engineering, BYU \\ Master of Science}

Fabrication of carbon infiltrated carbon nanotubes (CI-CNT) can result in large mechanical property variation, and methods to characterize properties usually involve destructive testing. Finding a non-destructive way to test for stiffness of this material reduces the number of parts that have to be made. It also simplifies testing of complex parts. The stiffness of CI-CNT beams is related to the type of carbon material infiltrated between the carbon nanotubes (CNTs), how it interacts with the CNTs, and how much of it there is. The amount of material can be estimated using the density of the beam, and both the type of material and its interaction with the carbon nanotubes can be approximated through analysis of the Raman spectra taken at the surface. A combination of these two observations can be related to the effective material stiffness. The relationship can be fitted with a power function, with a variance of $1.41 \mathrm{GPa}$, which is about $11 \%$ of the maximum stiffness of the samples tested. This variance is similar to the larger variations in CI-CNT beam stiffnesses found in a single batch of beams.

Keywords: Raman Spectroscopy, density, Carbon Nanotubes, CI-CNT, Bending Modulus, beam 


\section{ACKNOWLEDGMENTS}

I'd like to acknowledge the efforts of Shane Sypherd, Daniel Snow, and Andrew Cunningham in the creation, testing, and SEM imaging of the CI-CNT beams used in this study. I would also like to acknowledge the help of Dr. Patterson for assistance in using the Raman spectrometer. The input from Dr. Eric Homer, Dr. Richard Vanfleet, Alex Avila, and the BYU statistics office have been invaluable. I would also like to express appreciation for the time Dr. Brian Jensen spent to keep the project moving forward as well as reviewing parts of my thesis as the research progressed. This work could also not have been done without the funding from Tag Heuer 


\section{TABLE OF CONTENTS}

LIST OF TABLES $\ldots \ldots \ldots \ldots \ldots \ldots \ldots \ldots \ldots \ldots \ldots$ vi

LIST OF FIGURES $\ldots \ldots \ldots \ldots \ldots \ldots \ldots \ldots \ldots$ vii

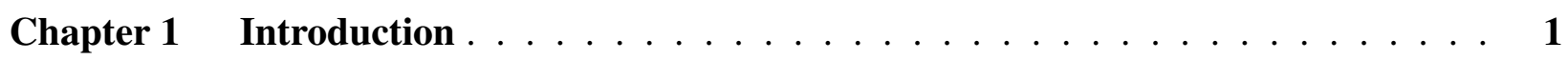

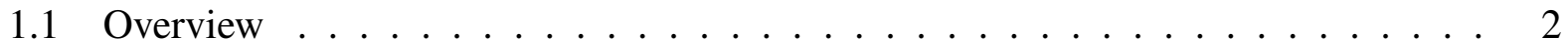

Chapter 2 Background . . . . . . . . . . . . . . . . . . . 4

2.1 Introduction . . . . . . . . . . . . . . . . . . 4

2.2 Raman Spectroscopy Background . . . . . . . . . . . . . . . . 4

2.3 Carbon Raman Studies . . . . . . . . . . . . . . . . . . . . . 6

2.3 .1 CNTs . . . . . . . . . . . . . . . . . . 7

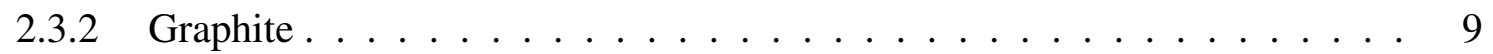

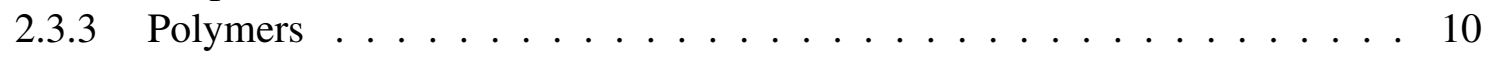

2.3.4 Amorphous carbon films . . . . . . . . . . . . . . . . 12

2.4 CI-CNT Raman . . . . . . . . . . . . . . . . . . . . . . . . . . 14

2.5 Additional Methods . . . . . . . . . . . . . . . . . . . . . . . . . . . . . . . . . .

2.6 Background Summary . . . . . . . . . . . . . . . . 16

Chapter 3 Research . . . . . . . . . . . . . . . . . . . 17

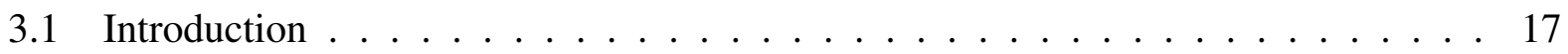

3.2 Methods . . . . . . . . . . . . . . . . . . 18

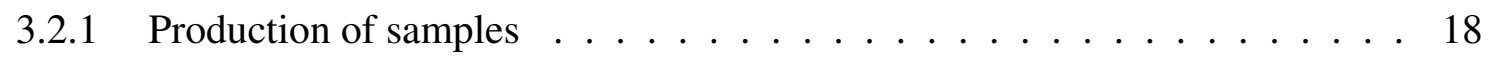

3.2 .2 SEM imaging . . . . . . . . . . . . . . . . . . . . 21

3.2 .3 Measuring Density . . . . . . . . . . . . . . . . . 22

3.2 .4 Raman Spectroscopy . . . . . . . . . . . . . . . . . . . . . 22

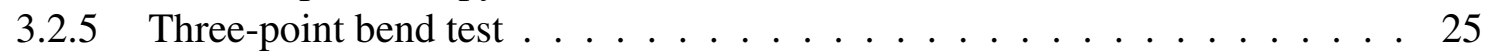

3.2 .6 Determining the relationship . . . . . . . . . . . . 26

3.3 Results . . . . . . . . . . . . . . . . . . . . 27

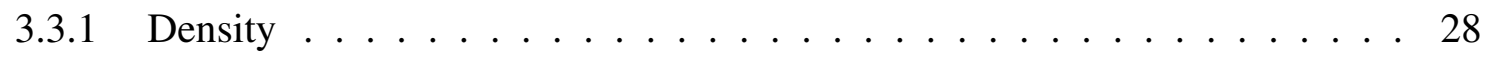

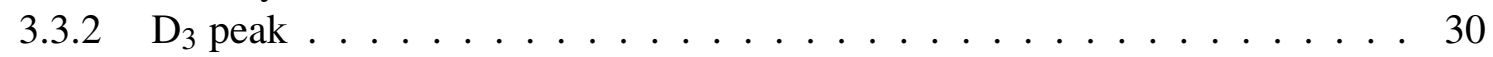

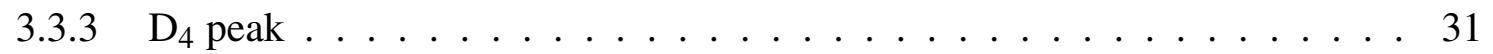

3.3.4 Raman parameters and growing conditions ............ 33

3.3 .5 SEM Images . . . . . . . . . . . . . . . . . . . . 33

3.3 .6 Bending Modulus . . . . . . . . . . . . . . . . . . . . . . . . . . . . . . 37

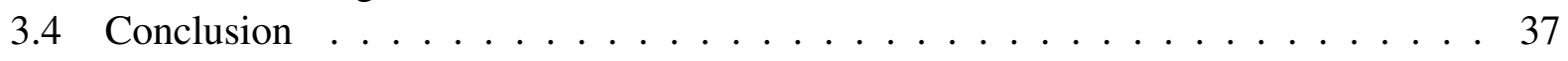

Chapter 4 Summary and Future Work $\ldots \ldots \ldots \ldots \ldots$

4.1 Results Summary . . . . . . . . . . . . . . . . . . . . . . . . . . . . . . . .

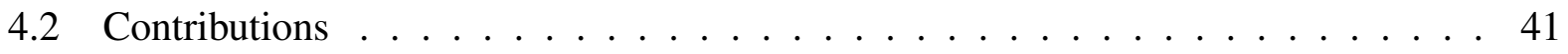

4.3 Future Work . . . . . . . . . . . . . . . . . . . . 42 


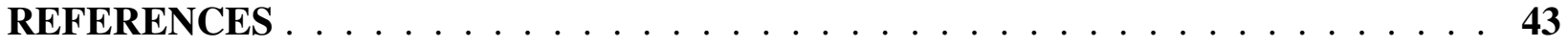

Appendix A $\operatorname{Raman}$ Spectrum fitting $\ldots \ldots \ldots \ldots \ldots \ldots \ldots$ 


\section{LIST OF TABLES}

3.1 Parameters used for growing CI-CNTs . . . . . . . . . . . . . . . . . 20

3.2 Parameters used for analyzing CI-CNTs . . . . . . . . . . . . . . . 26

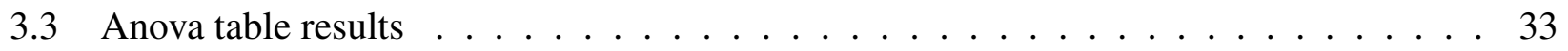

4.1 Material Properties of CI-CNT beams . . . . . . . . . . . . . . . . . . . . . . . . .

4.2 Peaks in CI-CNT Raman spectra . . . . . . . . . . . . . . . . . . . 41 


\section{LIST OF FIGURES}

1.1 An SEM image taken of the cross section of a CI-CNT beam . . . . . . . . . . . . 2

2.1 Plot of theoretical energy potential as seen in [1] and [2] with the continuum being the energy at seperation . . . . . . . . . . . . . . . . . 5 5

2.2 A Jablonski Diagram illustrating how a photon may interact with the energy states of a molecular structure (similar plots can be found in introductory Raman spectroscopy materials such as [2] and $[1] \ldots \ldots \ldots \ldots$

2.3 The key components of a Raman spectrometer are layed out . . . . . . . . . . . . 6

2.4 Raman spectra from [3] with three different source lasers as seen in the legend, the most intense being the highest frequency laser down to the least intense with the lowest frequency laser . . . . . . . . . . . . . . . . . . 8

2.5 Raman spectra from [4], CNTp is a spectrum of pristine CNTs while CNT3 and CNT15 are spectra of CNTs oxidized for 3 and 15 hours respectively . . . . . . . . . 8

2.6 Raman spectra from [5], SHER is highly ordered graphite with less impurities . . . . . 9

2.7 Raman spectra from [5], of HBC taken with a 633nm laser . . . . . . . . . . . . . 11

2.8 A grouping of carbon films based upon $\mathrm{sp}^{2}$ bonding, $\mathrm{sp}^{3}$ bonding, and hydrogen content $[6] \ldots \ldots \ldots \ldots \ldots \ldots \ldots \ldots$

2.9 An example of the Raman spectra of soot studied by Sadezky et al [5] . . . . . . . . . 13

2.10 An example Raman spectrum of CI-CNT beams from this study with each component of the fit labeled . . . . . . . . . . . . . . . . . . . . . 15

3.1 A zoomed in cutaway of the results from each step of process in making CI-CNT

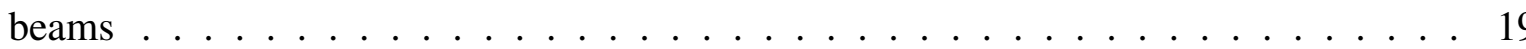

3.2 SEM image of a part of the cross section of a broken CI-CNT beam infiltrated at 311 sccm $H_{2}$ and $900^{\circ} \mathrm{C}$, for 25 min. . . . . . . . . . . . . . . . . . . . . . 21

3.3 Three Raman spectra of a single sample (sample 47) taken with the scan centered at the wavelengths in the legend. (a) shows uncalibrated spectra while (b) shows the affect of calibration in reducing amplitude uncertainty . . . . . . . . . . . . . 23

3.4 The rough solid curve is the raw data, and its fit is the smooth solid curve directly underneath (and partially obscured by) it. . . . . . . . . . . . . . . . . 24

3.5 Test fixture: the load was applied with a razor blade . . . . . . . . . . . . . . . . 25

3.6 Bending results from the 12 different beams in sample $142 \ldots \ldots$

3.7 The dashed line is the curve fit of the data, the dash dot curves are the $68 \%$ confidence interval, and solid green curves represent the $68 \%$ prediction interval . . . . . . . . 28

3.8 Two views of the measured data when plotted by the Raman parameters in equation 3.2 , the density, and the bending modulus. The blue line represents the two dimensional fit in figure $3.7 \ldots \ldots \ldots \ldots \ldots$

3.9 A surface plot of the relationship found between Raman spectra, density, and the bending modulus with the color turning red for higher modulus . . . . . . . . . . . . . 29

3.10 The top widths of columns inside CI-CNT beams measured with SEM versus the average density of the beams from the same sample . . . . . . . . . . . . . 30

3.11 SEM image of a part of the cross section of a broken CI-CNT beam infiltrated at 492.4 sccm $\mathrm{H}_{2}$ and $800^{\circ} \mathrm{C}$, for $70 \mathrm{~min}$. 
3.12 Example amorphous carbon Raman spectra from [6] illustrating the affects of bonding on the Raman spectrum . . . . . . . . . . . . . . . . . . . . . . . 32

3.13 Relationships between input and output parameters . . . . . . . . . . . . 34

3.14 Types of capping identified with SEM _ . . . . . . . . . . . . . . . 35

3.15 Model with SEM capped samples identified . . . . . . . . . . . . . . . . . . 35

3.16 Example of how a sample might be capped . . . . . . . . . . . . . 36

3.17 Model with samples infiltrated at $950{ }^{\circ} \mathrm{C}$ and $800{ }^{\circ} \mathrm{C}$ removed $\ldots \ldots \ldots . . . . .37$

3.18 Original model but with range of moduli in each sample point labeled . . . . . . . . 38 


\section{CHAPTER 1. INTRODUCTION}

Carbon infiltrated carbon nanotubes (CI-CNTs), a combination of CNTs and carbon films, have become an area of interest for 2 dimensional applications. This is because they can be patterned with microscopic accuracy and have a growth height that allows them to function at macrosizes without a substrate. Carbon nanotubes themselves (CNTs) have been explored for many one dimensional applications, and carbon films have been explored in protective coatings and in electronics. Researchers at Brigham Young University found a specific application for CI-CNTs in coronary stents because of the material's strength and biocompatibility. They found that CICNTs were resistant to corrosion and unlike many current implants, could be accepted by the body without additional layers [7]. Amorphous carbon films were also found to be bio compatible by exposing a culture of human cells to amorphous carbon [8].

Despite its many positive qualities, fabrication of CI-CNTs with repeatable properties can be difficult [7]. At the moment, designs must allow for large variation in material properties. Quality assurance can even be difficult as CI-CNT beams can have different sizes of voids and have a structure that changes as it approaches the surface (see Figure 1.1). In addition the chemical bond organization in the infiltrated carbon may change depending on deposition conditions, further changing the properties. Finding ways to limit variation in material properties between batches commonly involves destructive testing. These methods can be time consuming and expensive. This research describes a method to allow for cheaper, faster, non-destructive testing through Raman spectroscopy and density measurements.

Using non-destructive methods for quality control is already common in several materials. Some of the methods commonly used are mentioned here. Positive metal identification (PMI) or $\mathrm{X}$-ray fluorescence (XRF) have been made into handheld devices to identify various materials on

the factory floor. X Hou et al [9] found that handheld version of XRF can detect elements of solid materials fairly well despite its low sensitivity. This method is useful in metals as a quality control 


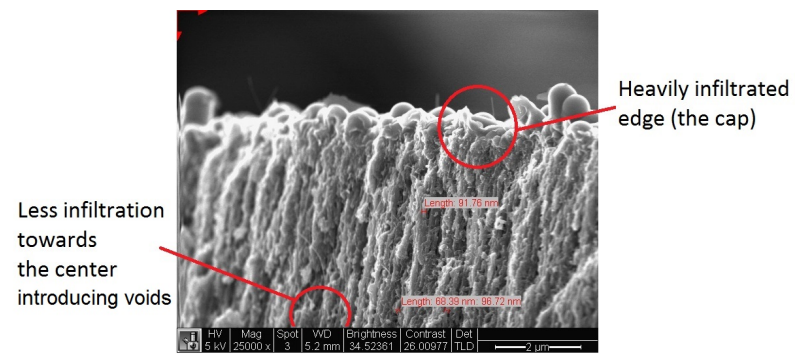

Figure 1.1: An SEM image taken of the cross section of a CI-CNT beam

method because it can determine the atomic make up of alloys. Another way to measure material properties is to relate the natural frequency of a beam to its geometry, density, and modulus of elasticity. Q. Zhao [10] et al used the natural frequency of small piezoelectric beams to illustrate the possibility of having sensors powered by vibrations within bridges. In the study both an analytical formula and FEA solutions were used to predict the observed natural frequency. The calculated beam frequencies were within $20 \%$ of the observed frequencies. This suggest a good estimate of a beam's stiffness can be made through measuring of its natural frequency. A third method is infrared (IR) spectroscopy, which has been used to identify materials in a similar manner to Raman spectroscopy. IR spectroscopy differs from Raman spectroscopy in that it measures absorption of light instead of shifts in light from a specific frequency, allowing different vibrational modes to appear [1]. Because of the uniqueness of materials in IR spectra, databases have been compiled of several different materials [11]. This allows an unknown sample to be identified by matching its IR spectra with one already recorded in a database.

While there are several methods of material identification, most methods have not been used to identify the stiffness of a specific sample. This study not only finds a way to identify CICNT beams but also estimates the bending modulus of individual beams. This study also helps better explain what CI-CNTs may be made of.

\subsection{Overview}

This chapter has been a brief overview of the problem being addressed in this thesis. The following chapters will discuss a method of predicting the effective bending modulus of CI-CNTs and what additional questions the method raises or leaves unanswered. 
Chapter 2 provides a brief background on the possible meanings of the different peaks in CI-CNT Raman spectra. The chapter also discusses possible materials that may make up CICNT beams. The information in this chapter provides needed insight into the conclusion made in chapter 3. Some of the alternate methods of identifying the structures in CI-CNT beams are briefly discussed. These methods may be used in future work to answer some of the questions in chapter 4.

Chapter 3 presents the findings of the thesis. In this chapter the methods used in preparing and testing the samples are clearly laid out, step by step. The data collected from the experiment are presented in this chapter as well as important trends. The predictor for the bending modulus of CI-CNTs is also given in this chapter.

Chapter 4 expands upon the concluding remarks in chapter 3. This chapter summarizes all of the results found in the study. The author's personal contributions are listed in this chapter as well. Future work needed to improve the prediction of the bending modulus with non-destructive testing will be briefly described. Additional questions from the study are made here as well as how they might be answered. 


\section{CHAPTER 2. BACKGROUND}

\subsection{Introduction}

Carbon infiltrated carbon nanotubes (CI-CNT) in this study were analyzed on a molecular level with Raman spectroscopy and compared with carbon materials in other studies.

While several methods can be used to study the material properties of CI-CNTs, Raman spectroscopy was chosen for its convenience and availability. The theory and methods used in Raman spectroscopy have been around since 1928 when Sir Chandrasekhra Venkata Raman first observed the phenomenon [2]. While the equipment and understanding has improved over the years, there are still basic concepts that remain universally used. Due to the similarities in standards used by researchers around the world, carbon materials such as nanocrystalline diamonds, graphene, and amorphous carbon can be compared through their Raman spectra. While additional methods in conjunction with Raman spectroscopy help better understand the spectra of a material, similar Raman features are often caused by the same mechanisms.

Additional methods such as X-ray flourescnece and molecular modeling have been used with Raman spectroscopy to better understand the structures of materials such as CNTs as well as why the spectra look the way they do. In this study SEM imaging, 3-point bending, optical measuring, and weighing of CI-CNTs were used to better understand CI-CNT beam stiffnesses but additional methods in future studies could provide more definitive answers to some of the questions answered with empirical methods.

\subsection{Raman Spectroscopy Background}

Raman spectroscopy is an optical method that allows materials to be identified by how they appear under laser light. To understand the theory behind Raman spectroscopy it is best to start with how atoms bond together. Figure 2.1 is based upon the plots found in both [2] and [1] and 


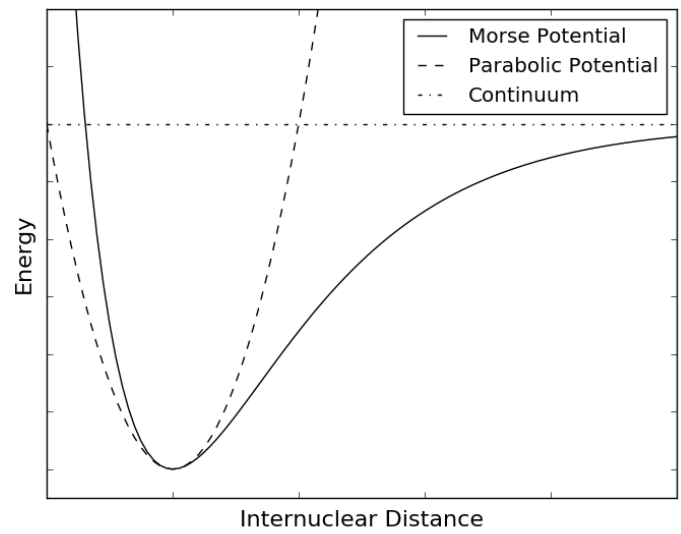

Figure 2.1: Plot of theoretical energy potential as seen in [1] and [2] with the continuum being the energy at seperation

shows the energy potential in atomic bonds. When atoms are too close together they repel one another but after a certain distance they become attracted once more. The stable bond distance is the lowest energy state. The two types of potentials in Figure 2.1 describes two different types of molecular vibrations. If the energy potentials were thought of as the potential energy in a spring then the parabolic potential describes harmonic vibrations, or oscillations caused by a restoring force linearly proportional to atomic displacement [1]. The Morse potential describes anharmonic vibrations [2] [1].

The resonant vibrations act as a subset in the electronic states in molecular structures. As seen in Figure 2.2 light hitting a molecule can do a few things. Normal light reflections such as what you would find in mirror are called Rayleigh Scattering (i.e. the same frequency of light sent to the sample bounces off the sample). Sometimes a certain frequency of light will cause a molecule to shift to an entirely new electronic state before releasing a high energy photon. This is called flourescence. The effect is usually very strong and can be seen with UV lasers on organic material. The very weak effects, Stokes and anti-Stokes, is what is used in Raman spectroscopy. This requires all the other affects to be filtered out from the spectrum.

A simplified example of a Raman spectrometer is given in Figure 2.3. For a good spectrum the sample should only be exposed to a single wavelength of light and the filter needs to filter out Rayleigh scattering. Using different types of source lasers can help reduce fluorescence in the 


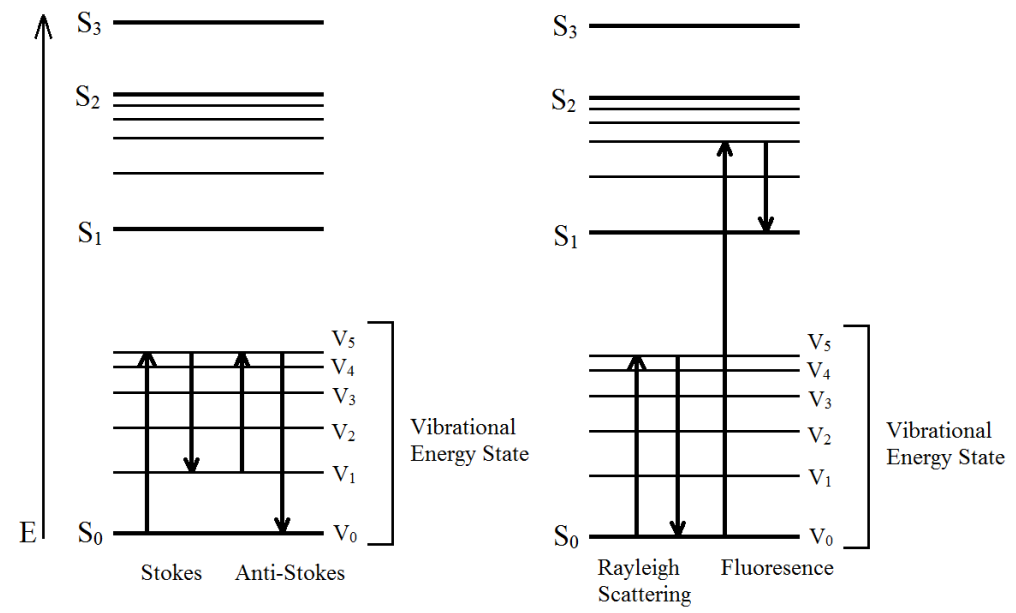

Figure 2.2: A Jablonski Diagram illustrating how a photon may interact with the energy states of a molecular structure (similar plots can be found in introductory Raman spectroscopy materials such as [2] and [1]

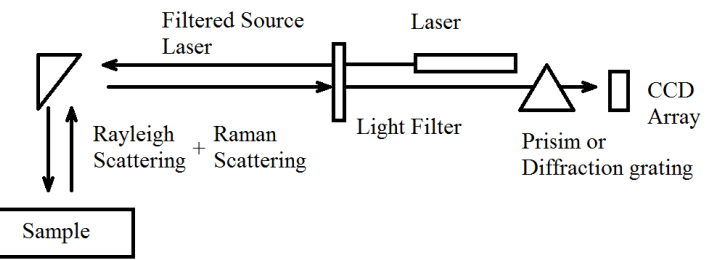

Figure 2.3: The key components of a Raman spectrometer are layed out

samples. A cooled CCD array is important to reduce noise caused by thermal energy from the room temperature air.

To consistently identify different aspects of a spectrum collected by the CCD array shifts from the original laser frequency are labeled with wavenumbers. A wavenumber is defined as 1/wavelength or the frequency divided by the speed of light [2]. While this isn't the same units as the frequency of light it is still conventional to say "frequency shift" [2].

\subsection{Carbon Raman Studies}

Throughout the study, research with different types of carbon were used to better understand the spectra of CI-CNTs under the assumption that CI-CNTs were carbon nanotubes (CNTs) 
infiltrated with a chaotic carbon structure. Four main types of carbon structures were looked at: CNTs, Graphite, polymers, and amorphous carbon films.

\subsubsection{CNTs}

CNTs can be found in several different conditions and Raman spectroscopy has been done on many of them; 3 examples will be mentioned here.

Multiwalled carbon nanotubes (MWCNT) were studied by Miroslaw Szybowicz et al [3] and M. Castriota et al [12]. The spectra they found was similar to that seen in Figure 2.4. Szybowicz et al related the D peak in Figure 2.4 specifically to defects and disorder in the carbon lattice. The $\mathrm{G}$ peak was found in all carbon materials reviewed by [3] and was associated by $\mathrm{C}-\mathrm{C}$ streching, in other words any $\mathrm{sp}^{2}$ carbon structures. It is interesting to see that the relative intensities of the $\mathrm{D}$ and $\mathrm{G}$ peaks depends on the source laser.

While M. Castriota et al used a much larger range of wavelengths they also used mainly two peaks to describe the spectra in the region given in Figure 2.4. A third peak called the L peak was also found in their study where MWCNTs had a carbon chain down the center of the tube. The carbon chain was suggested to be a carbine chain. The $\mathrm{G}$ band was found to change position position with the temperature of the MWCNTs. The study also expanded the spectrum to find that the $\mathrm{D}, \mathrm{G}$ and $\mathrm{L}$ peaks are first order resonance peaks since they found overtones of the $\mathrm{D}$ and $\mathrm{L}$ peaks at larger frequency shifts.

Single walled Carbon nanotubes (SWCNT) studied by M.S. Dresselhaus et al [13] have a very similar Raman spectra to MWCNTs in that they have both a D peak and a G peak. Unlike MWCNTs the D peak is very small in SWCNTs and the $\mathrm{G}$ peak splits into a $\mathrm{G}^{+}$and a $\mathrm{G}^{-}$peak. The $\mathrm{G}^{+}$peak at $1590 \mathrm{~cm}^{-1}$ represents the vibrations along the axis of the tube while the $\mathrm{G}^{-}$peak at $1570 \mathrm{~cm}^{-1}$ results from the circumferential vibrations in the SWCNTs. At $1754 \mathrm{~cm}^{-1}$ and 1775 $\mathrm{cm}^{-1}$ they found two additional peaks called $\mathrm{M}^{+}$and $\mathrm{M}^{-}$respectively, as overtones. Another small but important peak found in the study was at $1864 \mathrm{~cm}^{-1}$ and was assigned to the iTOLA vibration modes. This peak varied in position dramatically as the source laser wavelength changed.

S.A. Chernyak et al [4] oxidized CNTs in a concentrated $\mathrm{HNO}_{3}$ solution to see how it would affect the Raman spectra. As seen in Figure 2.5 they used 5 peaks (D1, D2, D3, D4, and G) to describe the spectra of oxidized CNTs. Only the D1 and G peak, similar to the MWCNT 


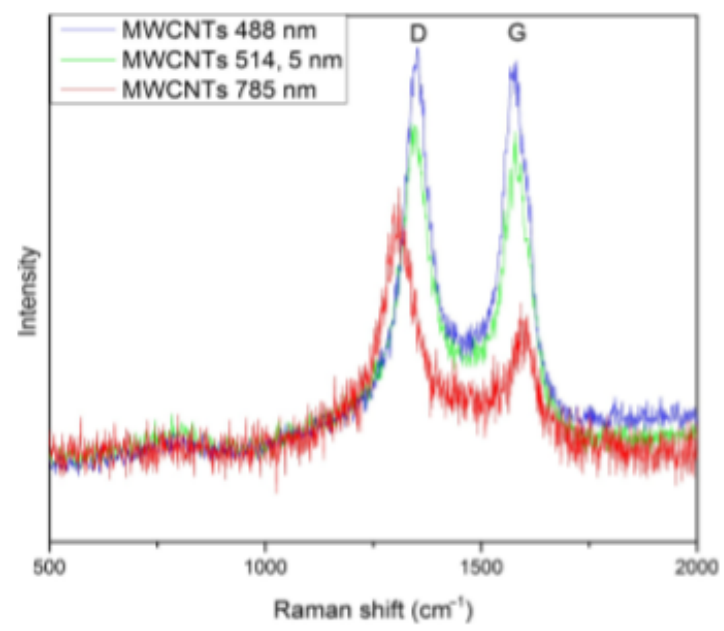

Figure 2.4: Raman spectra from [3] with three different source lasers as seen in the legend, the most intense being the highest frequency laser down to the least intense with the lowest frequency laser

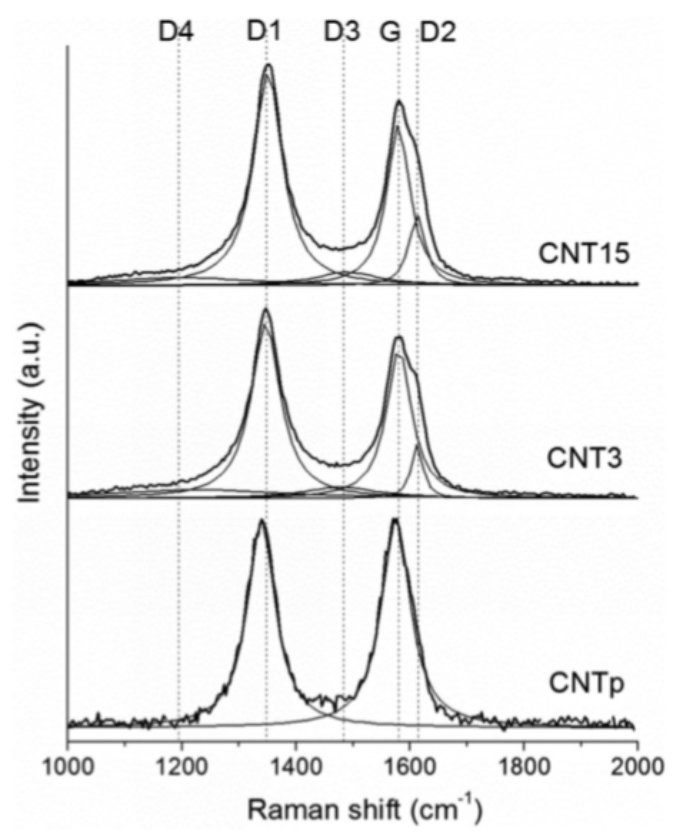

Figure 2.5: Raman spectra from [4], CNTp is a spectrum of pristine CNTs while CNT3 and CNT15 are spectra of CNTs oxidized for 3 and 15 hours respectively 


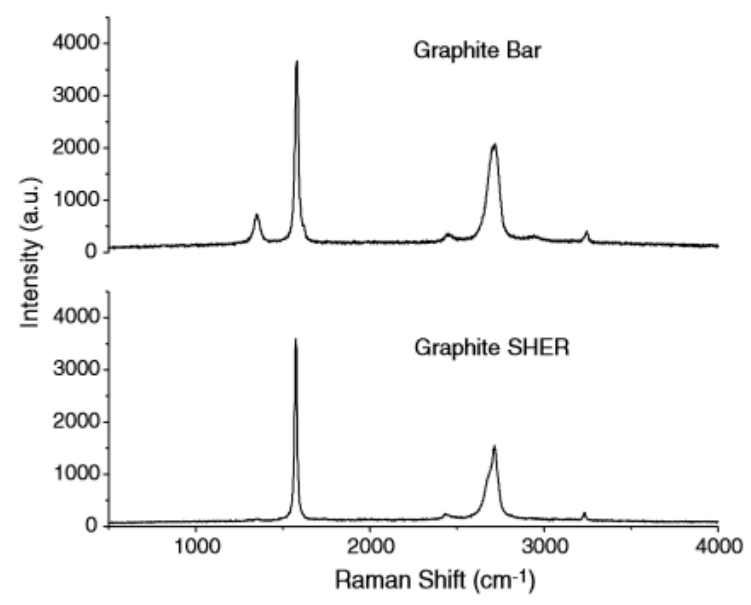

Figure 2.6: Raman spectra from [5], SHER is highly ordered graphite with less impurities

spectra, were needed to fit the pristine CNTs. The other peaks also grew in size at different rates during oxidation. The D2 band was attributed to vibrations of surface aromatic ring structures or edge carbon atoms. In other words breaking up the graphene lattice structure allowes for this vibrational mode. The D3 peak was assigned to defects in the stacking layers of graphene in the CNTs. The D4 peak was also explained in a few ways but was mainly attributed to polyene and polyphenylene fragments. From this example, and the previous two, CNT spectra can be described with a bare minimum of 2 peaks and additional peaks help explain any unique attributes.

\subsubsection{Graphite}

Large crystals of carbon come in mainly one of two ways: graphite sheets or diamonds. A Sadezky et al [5] used graphite as an example to better understand soot in their study. Figure 2.6 from the study shows two samples of graphite varying in purity. The tallest peak in the spectra is defined as the $\mathrm{G}$ peak and the peak immediately to its left (lower wavenumber) is defined as the D1 peak. In the more pure sample of graphite the D1 peak is all but gone. This illustrates why the $\mathrm{D}$ in the D1 peak usually stands for disorder or defects.

Microcrystalline graphite was studied by R.J. Nemanich and S. A. Solin [14]. Much of thier study involved trying to match a model of the vibrational modes of microcrystalline graphite with the actual Raman spectra. The study used four Lorentzian curves to fit the spectra. One curve was used for the background and the other three were centered at $1357 \mathrm{~cm}^{-1}, 1589 \mathrm{~cm}^{-1}$, and 
$1621 \mathrm{~cm}^{-1}$. The $1357 \mathrm{~cm}^{-1}$ peak was associated with disorder in the system from the crystal size instead of the defects in the crystals themselves. The other two peaks come from the splitting of the $\mathrm{E}_{2 g}$ mode, an in-plane vibrational mode. They found that the Raman spectra changed significantly as crystal size changed. They also found that established theoretical models they tried sometimes approximated the Raman spectra but still would inaccurately predict one or more Raman features.

J. Robertson [6] included nanocrystalline graphite in his review of amorphous carbon films. He also used density of states to help explain the structures of crystalline graphite. However, he only used two peak, a D peak and a G peak, to describe the Raman spectra. The D peak was defined as a breathing mode in aromatic rings. The $\mathrm{G}$ peak was associated with $\mathrm{C}-\mathrm{C}$ bonding. With these two peaks he found that the $\mathrm{I}_{D} / \mathrm{I}_{G}$ ratio was at a maximum in carbon films in nanocrystalline graphite and that the $\mathrm{G}$ peak could shift as far right as $1600 \mathrm{~cm}^{-1}$ by changing the crystal size.

Similar to J. Robertson's review, Andrea Ferrari and J. Robertson [15] studied several carbon films, among which was nanodiamonds. In the study they used two main peaks to describe the Raman spectra, a D and a G peak, as well as a $v_{1}$ and a $v_{2}$. The D peak at $1350 \mathrm{~cm}^{-1}$ and the $\mathrm{G}$ peak at $1550 \mathrm{~cm}^{-1}$ were associated with amorphous carbon. The peak at $1150 \mathrm{~cm}^{-1}, v_{1}$ and the peak at $1480 \mathrm{~cm}^{-1}, v_{2}$ were argued to belong to trans-polyacetylene instead of being related boundaries in nanodiamonds. In the study, changing the source laser made it easier to see the $v_{1}$ and $v_{2}$ peaks. From these studies of crystalline carbon structures, it can be seen that changing the size of crystal boundaries can introduce new materials or new peaks. Any defects in the crystal structure can also add more than one feature to the Raman spectra.

\subsubsection{Polymers}

While polymers are mainly associated with plastics they can sometimes show up in other carbon structures such as was found in the study of nanodiamonds [15]. Hexa-benzo-coronene (HBC) studied by A. Sadezky [5] was described by them as a graphene layer with carbon strings. As seen in Figure 2.7 this structure has a Raman signature significantly different then graphene or graphite. The spectrum was described by three peaks, a $\mathrm{G}$ peak at $1600 \mathrm{~cm}^{-1}$, a D peak at 1320 $\mathrm{cm}^{-} 1$ and another unnamed peak at $1250 \mathrm{~cm}^{-1}$. The paper compared these peaks to those found in soot. They found that the peaks had similar positions to peaks found in soot. This supported 


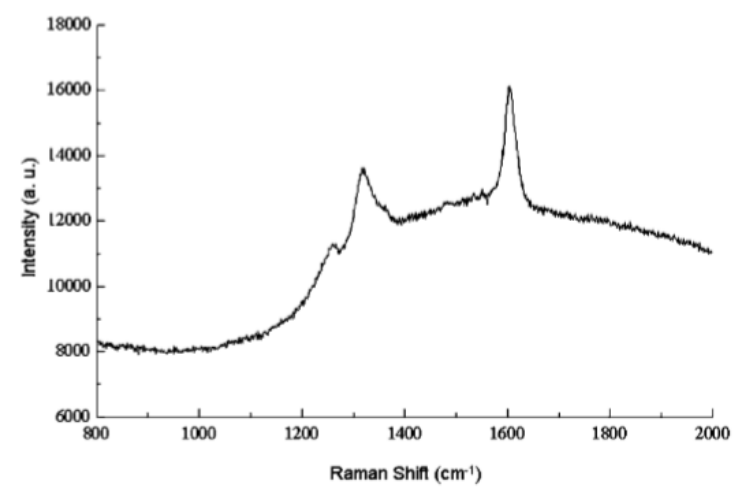

Figure 2.7: Raman spectra from [5], of HBC taken with a 633nm laser

using the HBC structure as a model for the basic structure of the graphitic domains in soot. The HBC structure itself was simple enough for them to analyze with theoretical models.

Peter J. Larkin [16] described the Raman peaks that can come from different molecular bonds. Of the bonds applicable to the study of CI-CNTs he lists two types of structures: ciscarbon chains, and aromatic rings. Cis-carbon chains were identified with a single peak in the range described in Figure 2.7 at $1630-1660 \mathrm{~cm}^{-1}$. A single aromatic ring had four given Raman active modes at $1585-1625 \mathrm{~cm}^{-1}, 1565-1590 \mathrm{~cm}^{-1}, 1470-1525 \mathrm{~cm}^{-1}$, and $1400-1465 \mathrm{~cm}^{-1}$. As described in his book, adding any sort of group, or substitution of any of the bonds would affect the vibrational modes, or even create new ones.

Teobald Kupka et al [17] studied polyenes and how their length can affect their vibrational modes. They specifically studied all trans and all cis carbon chains. Each carbon chain was described with two vibrational modes, one for the single bonded carbon and one for the double bonded carbon. The double bonded carbon peak was around $1360 \mathrm{~cm}^{-1}$, with the highest frequency shift in the middle of the chain and the lowest at the ends. The opposite was found with the single bonded carbon, which has a peak around $1440 \mathrm{~cm}^{-1}$ in all-cis carbon chains and $1430 \mathrm{~cm}^{-1}$ in all-trans carbon chains. These peaks shifted to lower wavenumbers when the chains got longer. The exception was the peak for single bonded all-cis carbon which shifted to higher wavenumbers instead. 


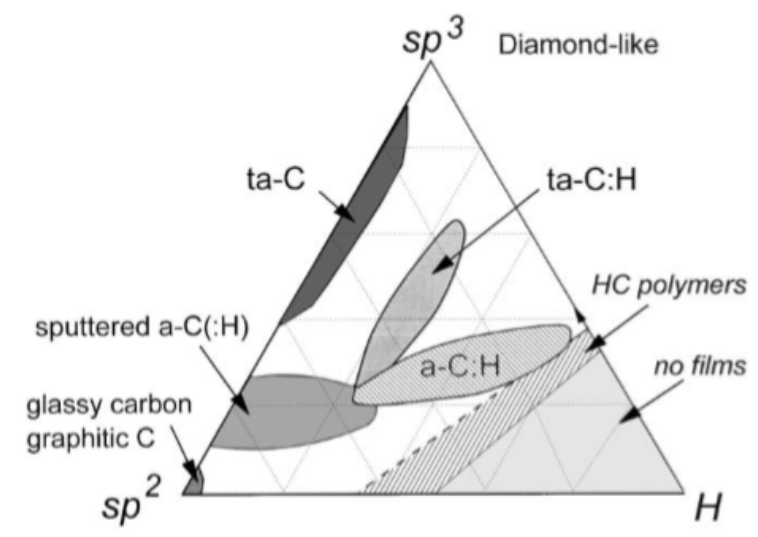

Figure 2.8: A grouping of carbon films based upon $\mathrm{sp}^{2}$ bonding, $\mathrm{sp}^{3}$ bonding, and hydrogen content [6]

Polymer-like carbon structures can have very specific peaks but as the structure grows in complexity these peaks can grow in number or width. Carbon chains for example have broader peaks as the chain grows longer.

\subsubsection{Amorphous carbon films}

Amorphous carbon films have been studied since the 1960's and over the year many different kinds of amorphous carbon have been identified. In Figure 2.8, a chart from [6], a continuum of carbon films is described. In addition to the films labeled we will discuss carbon structures in soot as are also very chaotic in their structures.

J. Robertson [6] did a review of amorphous carbon structures and used only two peaks, a $\mathrm{D}$ and a $\mathrm{G}$ peak to describe the main features of the Raman spectra. With two peak he was able to illustrate several relationships between types of carbon films and the position, or relative intensity of the $\mathrm{D}$ and $\mathrm{G}$ peaks. A notable exception is when he analyzed ta-C films which are known to have a larger fraction of $\mathrm{sp}^{3}$ bonding than other films. He used a third peak, $\mathrm{T}$ which was associated with $\sigma$ bond vibrations in the $\mathrm{sp}^{3}$ structures.

A. Sadezky et al [5] in studying soot used 5 different peaks and a linear background, as seen in Figure 2.9. The D1 and G peaks are associated with a graphite structure. The D3 peak was assigned to amorphous carbon structures such as organic molecules or fragments. The D4 peak was suggested to be polyene-like structures, carbon single or double bonds, or $\mathrm{sp}^{2}-\mathrm{sp}^{3}$ bonding. 


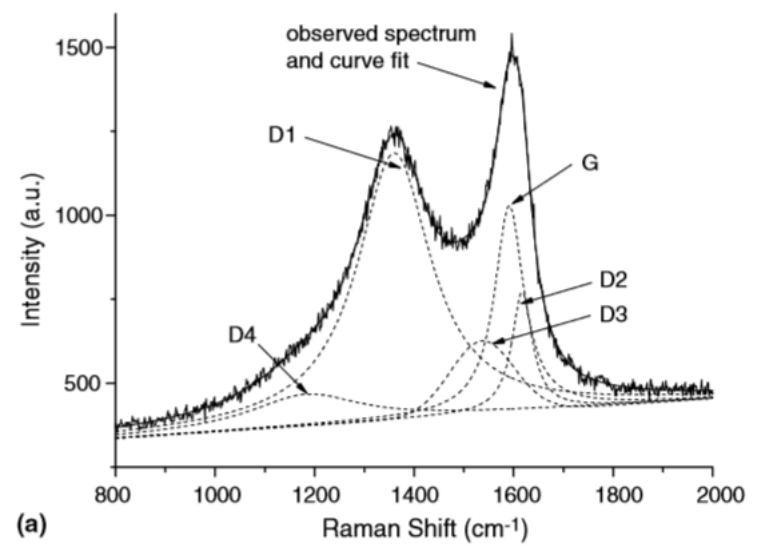

Figure 2.9: An example of the Raman spectra of soot studied by Sadezky et al [5]

Similar to [4] the D2 peak was assigned to be another vibrational mode in the graphitic lattice that seems to be suppressed in large lattice structures. While the number of the peaks seems to stay the same the intensities, as well as the background changed significantly when different source laser were used.

Matthew Smith et al [18] analyzed char with Raman spectroscopy and showed how 2-10 peaks could be used to describe the spectrum. As mentioned in their paper, using 10 peaks leaves some uncertainty in the fit to overfitting. The peaks in the range of $1250-1800 \mathrm{~cm}^{-1}$ were assigned to defects in aromatic rings, amorphous carbon, graphitic structures, and carbonyl stretching. Despite the large number of peaks used in the study they seemed to have correlated with the theoretical models. Using the 10 peaks in the study [18], they were able to find trends between the peaks and the kinds of char used.

Amorphous hydrogenated carbon (a-C:H) films have been studied by M. Veres et al [19], M. Rybachuck and J.M. Bell [20], and S.R.P. Silva et al [21]. [19] found three peaks in normal Raman spectra of a-C:H. The peaks were at $1394 \mathrm{~cm}^{-1}, 1557 \mathrm{~cm}^{-1}$, and $1610 \mathrm{~cm}^{-1}$. The paper showed how surface enhanced Raman scattering (SERS) can intensify features not normally found in the spectra. With SERS they found another peak at $1284 \mathrm{~cm}^{-1}$. [20] used 4 peaks in to fit the spectra of a-C:H. They identified the D and G peaks found in the previously mentioned studies as well as $\omega_{1}$ and $\omega_{3}$ peaks. They assigned these additional peaks to PPV and trans-carbon chains respectively. At higher source laser frequencies another vibrational mode labeled $\mathrm{T}$ appeared in their spectra centered at the lowest wavenumber. [21] looked at how a-C:H changed its spectrum 
as its hydrogen content changed. While still only using a D peak and a G peak like [6], they found that the D peak could shift as far as $1100 \mathrm{~cm}^{-1}$ which is on the lower end of the range given by the D4 peak in soot [5]. The dramatic shift of the D peak was associated with the change in the fraction of $\mathrm{sp}^{3}$ bonding.

In amorphous carbon films anywhere from 2 to 10 peaks have been used. This is in part because much of the chaotic structure of carbon films takes the form of polyemers or polyenes which each have their own unique vibrational modes. In general, a minimum of two peaks are needed to fit amorphous carbon films.

\subsection{CI-CNT Raman}

The CI-CNT structures studied in this thesis (see chapter 3) were scanned using Raman spectroscopy with a $785 \mathrm{~nm}$ laser. Figure 2.10 shows a sample Raman spectrum of from these scans. From the four types of materials listed above, the peaks in CI-CNT Raman spectra were identified. For example, the D1 and G peak came from the CNT studies since CI-CNT beams have CNTs in them. Amorphous carbon films and polyenes seemed to be a good fit for some of the features seen in CI-CNT beams, and seem to be as chaotic as the structure found between CNTs in CI-CNT beams. The Raman spectra of a CI-CNT beam shown in Figure 2.10 is very similar to the oxidized spectra of CNTs [4] as well as the soot [5] and have peaks similar to some of the materials just mentioned. The main difference between Figure 2.10 and Figure 2.5 is the Gaussian peak which could be coming from structures such as carbine as seen in [12] or some of the vibrational modes seen in [13].

While [4] was not confident in assigning amorphous carbon to the D3 peak, the research into amorphous carbon seems to apply to CI-CNTs. This is likely because of the similarities between the chaotic structure of the infiltrated material in CI-CNTs and amorphous carbon.The background was included to improve the fit in a similiar way as [21], [22], and [15].

After fitting the CI-CNT spectra the peak variations were compared to the Raman spectra of other carbon materials to ensure that a physical explanation existed even if we were not sure what the structure actually looked like on a molecular scale. 


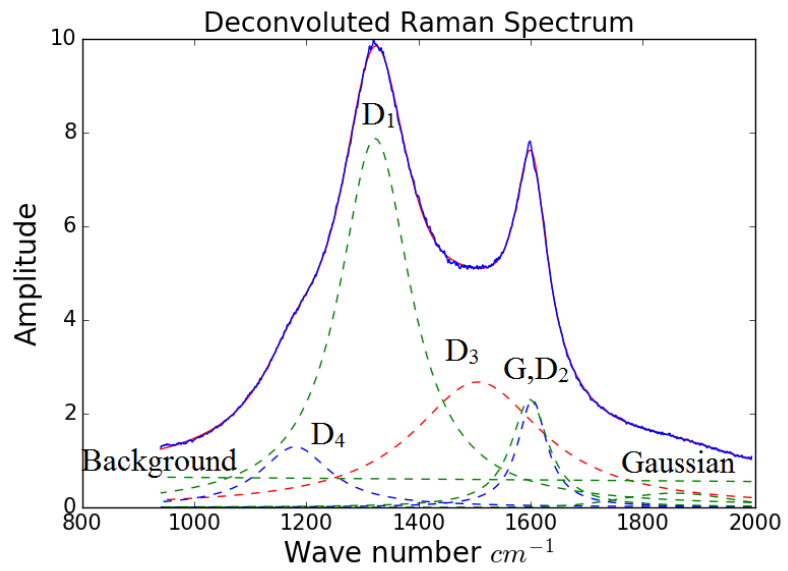

Figure 2.10: An example Raman spectrum of CI-CNT beams from this study with each component of the fit labeled

\subsection{Additional Methods}

Since so many different carbon materials have peaks assigned in the ranges of the peaks mentioned above it can be unclear what the CI-CNTs are actually made of. To determine what the peaks really mean in CI-CNT beams multiple methods of testing have to be done. Excluding Raman spectroscopy, methods that have been used to study carbon structures include x-ray diffraction (XRD), Neutron scattering, Nuclear Magnetic resonance (NMR) spectroscopy, and molecular modeling.

XRD has been used to characterize crystalline materials and thin films [23]. Samples are usually kept thin, as the thickness of the sample exponentially affects how much x-ray signal is absorbed [23]. XRD can provide information about the lattice structure of crystals, or the size of the crystal-like strutures in small particles such as soot [5]. The challenge with CI-CNT beams is that the structure is both a composite and it varies non-uniformly making it difficult to identify the peaks.

Inelastic neutron scattering has been used to study fullerenes [24]. This method uses neutrons that interact with the nuclei of atoms using only the nuclear force, thus being isotropic. The method is also limited to short durations of $10 \mathrm{~min}$ or less as the neutrons cool off over time and settle to the bottom of the chamber [25]. Similarly NMR, spectroscopy uses neutrons with a specific spin [25] or atoms such as carbon-13 also with spins. By vibrating the source atoms with 
some frequency, the atoms can interact magnetically to create various resonances depending on the material being tested [26].

Each of these methods provide different spectra and can give different kinds of information. All of these tests can be put together to filter out similar peaks, as multiple structures may be similar in Raman spectroscopy but look completely different in x-ray diffraction. By creating models, different theories can be tested by mathematically performing predictions of what peaks result when the models are tested with the various methods. Examples of using models to understand carbon structures can be seen in variety of works [13] [14] [27]. These models frequently take hours to test and take a thorough understanding of bonding energies to create. In the papers mentioned above many of the models created only approximate what the structure is as they don't always accurately predict the observed peaks.

Using several different methods can help narrow the possible meanings of various peaks but in addition to time and expense they need to be used correctly as each method has different sources of error. In this study, how peaks change with stiffness is compared to how stiffnesses of different materials, with similar peaks, change. While there is still uncertainty in the peak meanings it does provide insight into the structure of CI-CNTs.

\subsection{Background Summary}

CI-CNT beams are complex carbon structures that can only be understood thoroughly by using multiple kinds of tests. CI-CNT beams have unique Raman signatures that vary with their properties. The various peaks in CI-CNT Raman spectra can be compared to several different carbon materials to better understand their meanings. However, two or more methods, such as the ones mentioned in the previous section, are usually required to confirm the exact meaning of Raman peaks. Correlations can then be made through comparison of the results from the different methods. Comparisons such as those mentioned above have been used to determine the molecular structure of common carbon structures such as graphite, CNTs, and fullerenes. In this study the limited understanding of the meaning of the peaks is sufficient to relate Raman spectra and density with CI-CNT bending Modulus. 


\section{CHAPTER 3. RESEARCH}

\subsection{Introduction}

Materials used to create microscopic devices are much more limited than materials available to create macro-sized objects. Carbon infiltrated carbon nanotubes (CI-CNTs) are a unique material that can be useful in the microscopic world. Diamond-like carbon (DLC) films are known for being nonreactive, which is an important attribute for many mechanisms operating in high pressure or corrosive environments [28]. Carbon nanotubes (CNTs) themselves are fairly stable as well but can be reactive to some chemicals depending on their chirality [29]. CI-CNTs are in someways a combination of these two special materials giving it great potential as a new emerging material.

CI-CNTs are already being used in several ways in research. CI-CNTs can be used to make microscopic structures with high aspect ratios [30]. They have been used to create micro structures that are superhydrophobic [31]. Pyrolitic carbon and other similar carbon structures to CI-CNTs have been found biocompatible with animal cells. As a result CI-CNTs can be made as a stent that is more biocompatible then typical metals [7]. Micro-Electo-Mechanical-Systems (MEMS) devices that actuate with much less input force than their silicon counter parts [32]. As seen in several studies, material properties can be customized over a large range. The Young's Modulus can be varied from less than $1 \mathrm{GPa}$ to more than $15 \mathrm{GPa}$ and tensile strength from less than $1 \mathrm{MPa}$ to above $240 \mathrm{MPa}$ [33]. Geometry can also be controlled by growth time and patterning.

It is important to measure CI-CNT mechanical properties for several reasons. In MEMS, the stiffness of the material plays a significant role in how the mechanisms behave. MEMS devices have increasingly been made up of compliant mechanisms which frequently rely on large deflections [34] and CI-CNT beams can be made with very low stiffnesses. Several things affect the outcome of CI-CNT material properties. Changing the iron thickness can affect stiffness and strength but it also affects growth height [33]. Changes in growth and infiltration times will affect the height of the beams and how dense they are [33]. Changes in source gases were seen in 
this study to affect CI-CNT beam stiffnesses, but other studies show how it can affect the carbon structure of CNTs [35]. In amorphous carbon, the likely material infiltrating the CNTs in CI-CNT beams, changes in temperature or ion energy can change $\mathrm{sp}^{3} / \mathrm{sp}^{2}$ fractions, resistivity, and even density [6].

This study highlights what parts of the CI-CNT beams have the greatest effect on CI-CNT stiffness. By examining different parts of the Raman spectra and the density, some conclusions are made as to what makes a CI-CNT beam stiff. This study provides a non-destructive way to estimate the Young's Modulus perpendicular to the growth direction of CI-CNT beams. A model is then created to predict the stiffness of CI-CNT beams from density measurements and Raman spectra. This study also adds to the growing body of information on CI-CNT material properties, specifically the range of Young's Modulus achieved with chemical vapor deposition (CVD) grown CI-CNT beams. The following sections will describe how the study was conducted and what conclusions were made with the final model.

\subsection{Methods}

The procedures used during this study are based upon standard practices and methods. The following subsections describe sample preparation, sample testing, data organization, and data analysis.

\subsubsection{Production of samples}

The process to grow the CI-CNTs is based upon the process used by Jones et. al [7]. The summary of the procedure is summarized in 7 steps as follows and portrayed in Figure 3.1.

Step 1: Deposit thin layer of patterned photoresist on silicon wafer.

Step 2: Deposit $40 \mathrm{~nm}$ layer of alumina (prevents iron diffusion into silicon and influences the geometry of iron droplets [36]).

Step 3: Deposit a $4 \mathrm{~nm}$ layer of iron (serves as catalyst for growing carbon nanotubes).

Step 4: Sonicate the wafer with NMP to 'lift off' the patterned photoresist and the layers of iron and alumina directly above it, leaving behind patterned iron and alumina. 


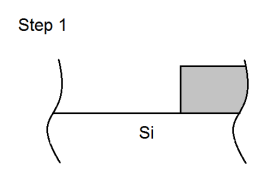

Step 3

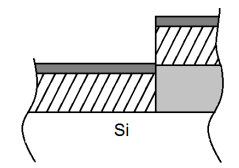

Step 5

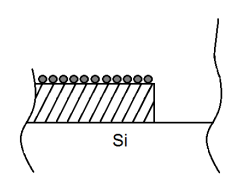

Step 7

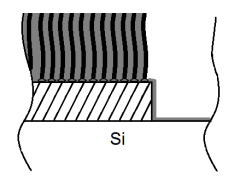

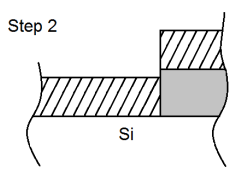

Step 4

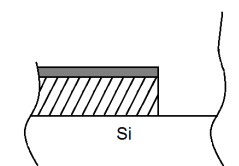

Step 6

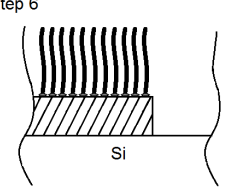

VID = Alumina

$=$ Iron

IIII = Carbon Nanotubes

III = Infiltrated Carbon Nanotubes

Figure 3.1: A zoomed in cutaway of the results from each step of process in making CI-CNT beams

Step 5: Reduce the iron film with hydrogen gas at an elevated temperature (this step was completed while the furnace heated up).

Step 6: Run pyrolytic carbon from decomposed ethylene at $750{ }^{\circ} \mathrm{C}$ across the surface to grow the nanotubes.

Step 7: Infiltrate the carbon nanotube forest with pyrolytic carbon by flowing ethylene mixed with hydrogen at $800^{\circ} \mathrm{C}$ or higher.

The study focuses on changing step 7 to produce CI-CNT beams with different stiffnesses. By keeping all the other steps unchanged all the CI-CNT beams begin with a similar CNT forest. Step 7 was repeated with 4 different temperatures, 4 different infiltration times, and 3 different hydrogen flow rates. Table 3.1 lists the parameters used. The three-factor study resulted in 48 (4 temperatures $\times 4$ times $\times 3 \mathrm{H}_{2}$ flow rates) possible infiltration combinations with 3 replicates per combination. 
Table 3.1: Parameters used for growing CI-CNTs

\begin{tabular}{|c|c|c|c|}
\hline Time $(\mathrm{min})$ & Temperature $\left({ }^{\circ} \mathrm{C}\right)$ & $H_{2}(\mathrm{sccm})$ & $\mathrm{C}_{2} H_{4}(\mathrm{sccm})$ \\
\hline 10 & 800 & 311 & 338 \\
\hline 25 & 850 & 396.5 & \\
\hline 45 & 900 & 492.4 & \\
\hline 70 & 950 & & \\
\hline
\end{tabular}

The beams were patterned to be about $.2 \mathrm{~mm}$ by $6 \mathrm{~mm}$. The CNTs were grown for the same time for each batch keeping the height between $.2 \mathrm{~mm}$ and $.7 \mathrm{~mm}$. The beams needed to be long enough for significant deflection for bend testing. It was also preferred to have the CNTs to be taller than the width of the beam to make it easier to put the beam on its side during testing. The small size allowed for several beams to be grown in one batch with consistent properties across each beam. Larger beams increase the likelihood of defects as any spec of dust at any point in the process can leave a patch that prevents CNT growth.

The entire process was done in the same high temperature tube furnace for each sample. The tube furnace had an inner diameter of $2.54 \mathrm{~cm}$ and a heated length of $30.48 \mathrm{~cm}$. The furnace used mass flow controllers (MFCs) to achieve target flow rates accurate within $0.4 \mathrm{sccm}$.

During the study some combinations were removed because of difficulty with production. Samples infiltrated for 10 minutes at $800{ }^{\circ} \mathrm{C}$ failed to be testable. All samples infiltrated for 70 minutes at $950{ }^{\circ} \mathrm{C}$, and samples infiltrated at $950{ }^{\circ} \mathrm{C}$ with $311 \mathrm{sccm}$ of $\mathrm{H}_{2}$ had difficulty producing testable samples but produced a few testable samples that were included in the study. The failed samples were too brittle to remove from the substrate, or they would shatter in the tube furnace while growing. Some samples grown at $800{ }^{\circ} \mathrm{C}$ for 10 minutes would compress significantly when attempting to remove them from the substrate for testing. Some samples did not have three replicates because of issues like those mentioned above, although one or two samples were still tested. Over 100 batches were successfully grown and tested, providing data over the range of the desired parameters. 


\subsubsection{SEM imaging}

Scanning Electron Microscope (SEM) imaging allows a visual analysis of the material inside the samples to better understand the relationship between the CI-CNT carbon structure and its stiffness in future samples. The method for preparing samples for SEM imaging was as follows. A beam taken from a given sample was broken by pressing a razor blade across the beam. The broken beams were fixed to a fixture angled at $45^{\circ}$ to allow easy viewing of the beams' cross sections. The beams were attached to the conductive fixture with carbon tape. The carbon nanotubes being either conductive or semi-conductive, were successfully imaged without an additional conductive layer [37].

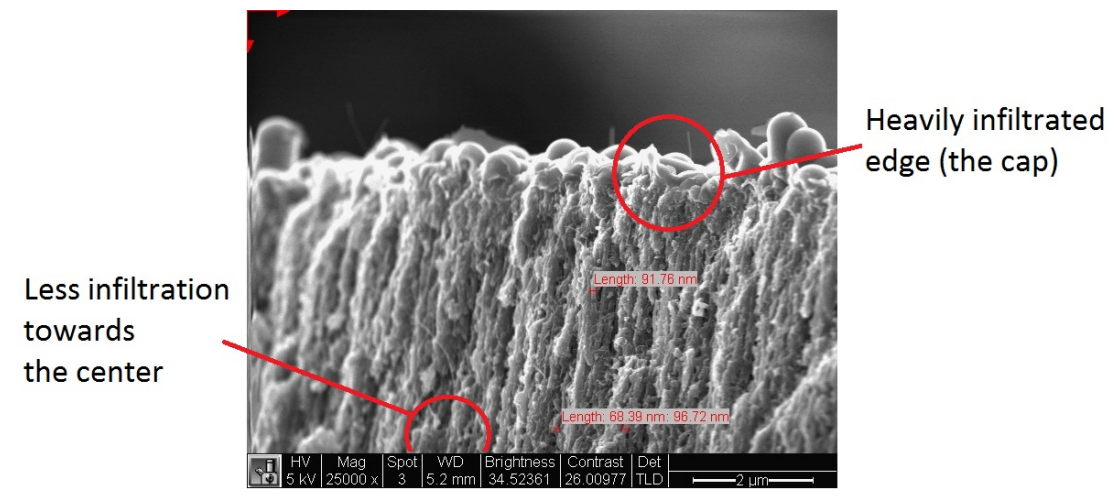

Figure 3.2: SEM image of a part of the cross section of a broken CI-CNT beam infiltrated at 311 sccm $\mathrm{H}_{2}$ and $900^{\circ} \mathrm{C}$, for $25 \mathrm{~min}$.

SEM images were used to estimate diameters of infiltrated CNTs, and whether the sample was "capped". Diameter measurements were taken at 6 different points, 3 at the top of the crosssection and 3 at the bottom of the cross-section. We defined capping as the state when pillars of carbon nanotubes merged together near the top of the cross-section and formed a different microstructure than the rest of the body. Capping was determined subjectively using SEM imaging and so some samples may be misidentified as capped. In other words another researcher may interpret some of the capped samples in this study as not capped since the definition of capping can be vague in some samples. Figure 3.2 shows an example of a sample that is capped. Due to the expense of SEM imaging, not all replicates for each combination of settings were investigated with SEM. 


\subsubsection{Measuring Density}

Measuring density is the first non-destructive test in the study, and it can be used to estimate how infiltrated a sample is. The density of CI-CNT beams from each sample was found using a batch method. Individual CI-CNT beams were too light to provide an accurate weight measurement. To decrease the noise of the scale, 12 beams from one sample were weighed together on a scale with $.0001 \mathrm{~g}$ accuracy. With 12 beams, the weight was recorded with 2-3 significant digits. The dimensions of individual beams were also optically measured with a microscope to the nearest micron. The average density for each batch was given by dividing the measured mass of the entire batch by the total volume of the batch.

Density was chosen to measure the interior of the CI-CNT beams because of its simplicity. The interior of the beams is difficult to view externally because the beams are too thick for many optical methods. Raman Spectroscopy, for example, only penetrates a few microns to several nanometers of the surface, while SEM imaging penetrates even less. Vibration analysis of beams may be another alternative to estimating stiffness, but the beams can be very fragile. Since the density measurement averages the entire sample, each sample will only have one data point from each test.

\subsubsection{Raman Spectroscopy}

Raman spectroscopy is the second non-destructive test that allows the type of infiltration to be quantified by looking at the surface. Aside from not damaging the beams, Raman spectroscopy was chosen as the method to test the surface of the beams because of the amount of information it provides for a low cost [1]. This study used a $785 \mathrm{~nm}$ laser, which provided a clear spectrum of each sample with a relatively low intensity beam, reducing the chance of oxidizing the surface [38]. Raman provides information about the vibrational behavior of the matrix of the material instead of the atoms themselves [1] [39]. Raman spectroscopy was also chosen because many bulk materials have been identified by their Raman spectra [40] [11]. Many forms of carbon have been studied with Raman spectroscopy including fullerenes [41], diamond-like amorphous carbon films [6], and CNTs [4]. 
Raman Spectroscopy is commonly used to look at an individual spectrum or the wave lengths of different peaks; since peak amplitudes will be compared in this study it is important that the spectrometer is calibrated correctly. The Renishaw inVia Raman Spectrometer used in this work is designed to be calibrated for peak positions relative to some known spectra such as silicon or neon. However, in this study, relative amplitudes are crucial, so data was also corrected by using standard calibration methods for CCD arrays which can be found in Mclean et. al [42]. The biased dark current signal was removed by taking the median at each wavelength of 99 separate scans. The scans were taken for 5 minutes each with an initial wait time of 30 minutes to allow for cooling. The flat-field image in this study was done by sweeping a neon peak across the CCD array at 100 different pixels, 9 different times. The charged bias image was taken in 200 scans with each scan taking 0.1 seconds. The overall effect of this additional calibration can be seen in Figure 3.3.

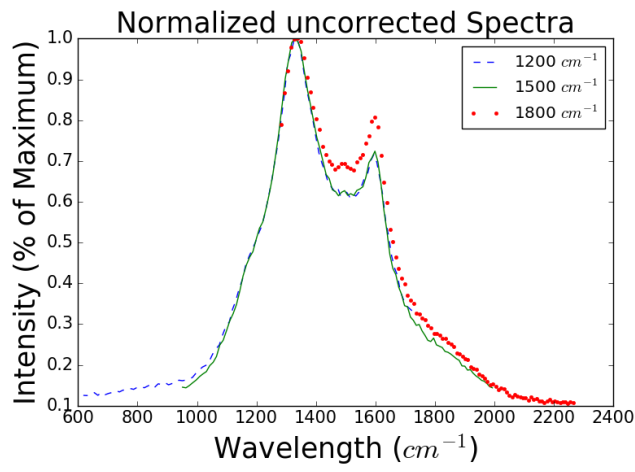

(a) (a)

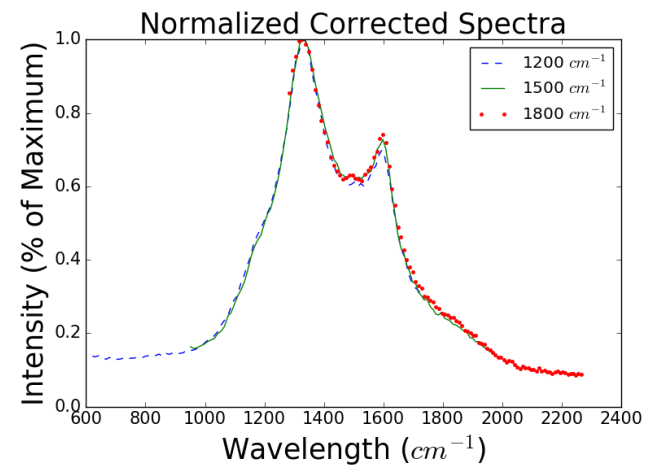

(b) (b)

Figure 3.3: Three Raman spectra of a single sample (sample 47) taken with the scan centered at the wavelengths in the legend. (a) shows uncalibrated spectra while (b) shows the affect of calibration in reducing amplitude uncertainty

The Raman spectra of the CI-CNT beams was prepared for fitting by dividing the entire spectra by the max intensity and then multiplied by 10. The spectra were broken into 6 different peaks (see Figure 3.4). Each peak was fitted with either Lorentzian or Gaussian peaks based upon research done on carbon materials that seem to be similar [4] [14] [5]. Five Lorentzian shaped curves were used for the 5 labeled parts in figure 3.4. One Gaussian curve was used as a place holder that represents region of figure 3.4 that is not typically defined in carbon structures. The 


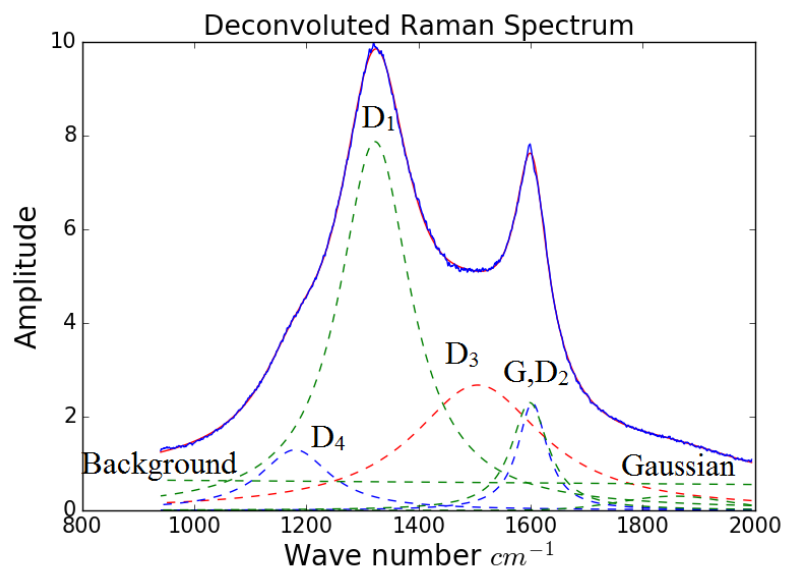

Figure 3.4: The rough solid curve is the raw data, and its fit is the smooth solid curve directly underneath (and partially obscured by) it.

Gaussian curve represents several potential curves that may or may not be present in this study and have not been well defined in research. Peaks were expected to shift small amounts due to tension in the sample or changes in the molecular structures [43]. During fit of the peaks, the $D_{2}$ peak was artificially limited to be shorter and to the right of the $\mathrm{G}$ peak because it usually represents disorder in the graphitic lattice [5].

Some peaks have been combined or subdivided in other research, ( [6] [44] just use two peaks while [45] uses 9 peaks) but for the sake of the fit in this study, the peaks were limited to 6 to prevent overfitting. Overfitting was briefly checked by obtaining similar fits from different initial conditions. The peaks initial positions were chosen by using peaks commonly found in multiwalled carbon nanotubes (MWCNTs). The Gaussian curve was added as the 4 peaks used in other research (D,G,D',D2) did not describe it. The peaks were adjusted with a linear optimizer commonly known as lmfit until they described the spectra found in CI-CNTs. With the linear background the total number of variables was 27. By quantifying the Raman Spectra, a model could be developed without fully understanding what every CI-CNT beam had deposited on its surface. 


\subsubsection{Three-point bend test}

The three-point bend test provides a simple way to obtain the bending modulus and ultimate strength of CI-CNT beams. While this study only bends the beams in a certain direction, other three-point bend tests have been done on CI-CNTs [33]. The setup of the test in this study followed the general guidelines in standard practice [46]. The test fixture setup can be seen in Figure 3.5. Beams were centered on the fixture by eye. The bending modulus was estimated using the straight portions of each curve (see Figure 3.6). The relationship of the force displacement curve to the modulus is given by $E=\frac{F}{\delta}\left(\frac{l^{3}}{48 I}\right)$ (see [47]). The geometry is captured by the second moment of area I, the displacement is given by $\delta$, the distance between supports by $l$, and the force exerted by F. The curve, which starts just before contact, at the beginning of each data set was ignored as directed by the standard practice [46].

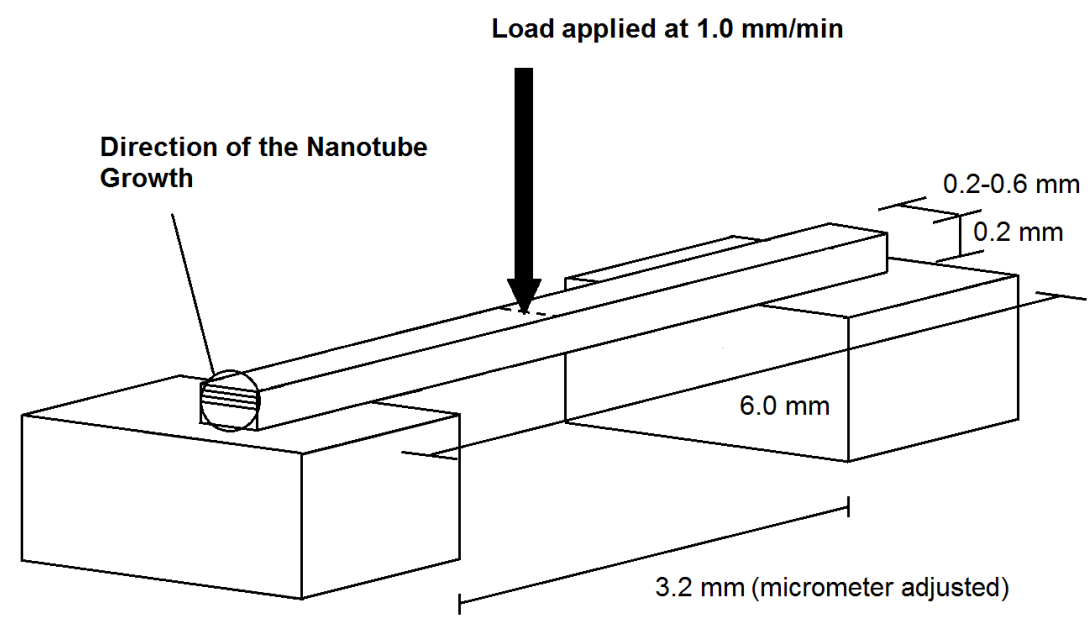

Figure 3.5: Test fixture: the load was applied with a razor blade

The bending modulus was identified by taking the average of several beams from a given sample. Each displacement plot was inspected and trimmed by hand for a linear fit to determine the modulus. The trimming was done by hand due to random linear portions in the part of the forcedisplacement curves to be removed. The cross-section was determined from optical measurements of each beam. The beams were treated as perfect rectangular prisms. The beams were brittle enough to assume yielding was negligible. 


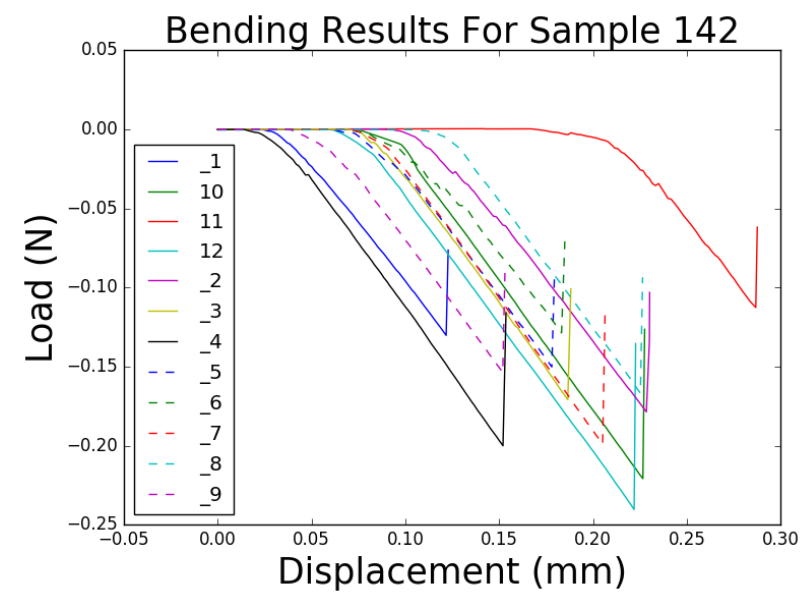

Figure 3.6: Bending results from the 12 different beams in sample 142

\subsubsection{Determining the relationship}

Table 3.2: Parameters used for analyzing CI-CNTs

\begin{tabular}{|c|c|c|}
\hline Property & Parameter 1 & Parameter 2 \\
\hline D1 & amplitude & position \\
\hline D2 & amplitude & position \\
\hline D3 & amplitude & position \\
\hline D4 & amplitude & position \\
\hline G & amplitude & position \\
\hline Gaussian & amplitude & position \\
\hline Background & slope & y-intercept \\
\hline density & mass per volume & \\
\hline
\end{tabular}

The empirical relationship was found by multiplying or dividing different combinations of density and Raman spectrum properties, such as peak height and wavelength. The parameters considered can be seen in table 3.2. Every combination was then tried up to 6 different parameters in a single combinations leading up to 14,684 different combinations in which a parameter could be multiplied or divided by other parameters. Essentially all the data collected that described the Raman spectra and the density of the beams were initially treated equally. The each data set was also scaled by dividing the entire $\mathrm{x}$-axis by the maximum value found in the current combination to assist in the optimization. The final formula was scaled back afterwards. To reduce overfitting the 
Baysian information criteria (Penalty $=n \times \log \left(\sigma^{2}\right)+k \times \log (n)$ ) given by [48], was used to add a penalty for every additional property used in the combination instead of the least squares fit. The penalty uses the number of data points (n) and the number of variables $\mathrm{k}$, as well as the variance

of the fit $\sigma$. In other words any group of 4 parameters would have a larger added penalty than any group of 3 parameters. The fitness of a combination was determined by how well a power model fit the data. The Raman spectrum properties in a combination were then multiplied by a power that reduced the error in the fit. This power was also found by optimization as too high or too low of a power and the fit worsened but somewhere in between it would improve. This helped to treat the Raman as a different type of parameter then density as they result from completely different tests.

A power fit was chosen because the model has no negative values and can be treated as a straight line in a log-log plot. A second order polynomial doesn't fit the final model nearly as well as a power fit. Increasing the order of a polynomial to obtain a similar variance would also increase the chances of over fitting the data. The overall model used can be seen in equation 3.1 where $\mathrm{x}_{1}$ is density and $\mathrm{x}_{2}$ is some combination of Raman parameters. The variables $a, c$, and $d$ are constants determined during the first optimization and do not change during the Raman parameter optimization. Variable $b$ is a constant determined during the optimization of the fitting the Raman properties to the have the power that produces the best fit.

$$
E=a\left(x_{1} x_{2}^{b}\right)^{c}+d
$$

\subsection{Results}

Based on the evaluation of each possible Raman term, the relationship between the Raman Spectroscopy, density, and the effective bending modulus was found and is given in figure 3.7 and equation 3.2.

The relationship shows that the Raman properties play a smaller role in the stiffness of the material than density. The effect of the Raman parameters in the empirical relationship is demonstrated in figures 3.8 and 3.9. The points in the scatter plot go from blue to red as the bending modulus increases. Raman or density alone does not tell a complete picture, and it is multiple parts of the Raman spectra that affect stiffness. Together, the model has a variance of $1.41 \mathrm{GPa}$. The model has a $68 \%$ or one standard deviation confidence interval of $2.1 \mathrm{GPa}$. This simply reflects 
how confident we can be of the fit. Increasing the number of data points decreases this interval. The 4.9 GPa prediction interval for the model provides $68 \%$ or one standard deviation confidence that any future sample will lie some where in its interval. This reflects both our confidence in the fit and the spread of the data. An infinite data set may still have a large prediction interval. Some of the sources of error will be discussed in the following sections.

Elastic modulus $(\mathrm{GPa})=1.3351 \times 10^{-4}\left(\rho\left(\frac{D 3_{a m p} D 4_{a m p} D 4_{c e n}}{D 3_{c e n}}\right)^{.75}\right)^{1.3435}-0.2485$
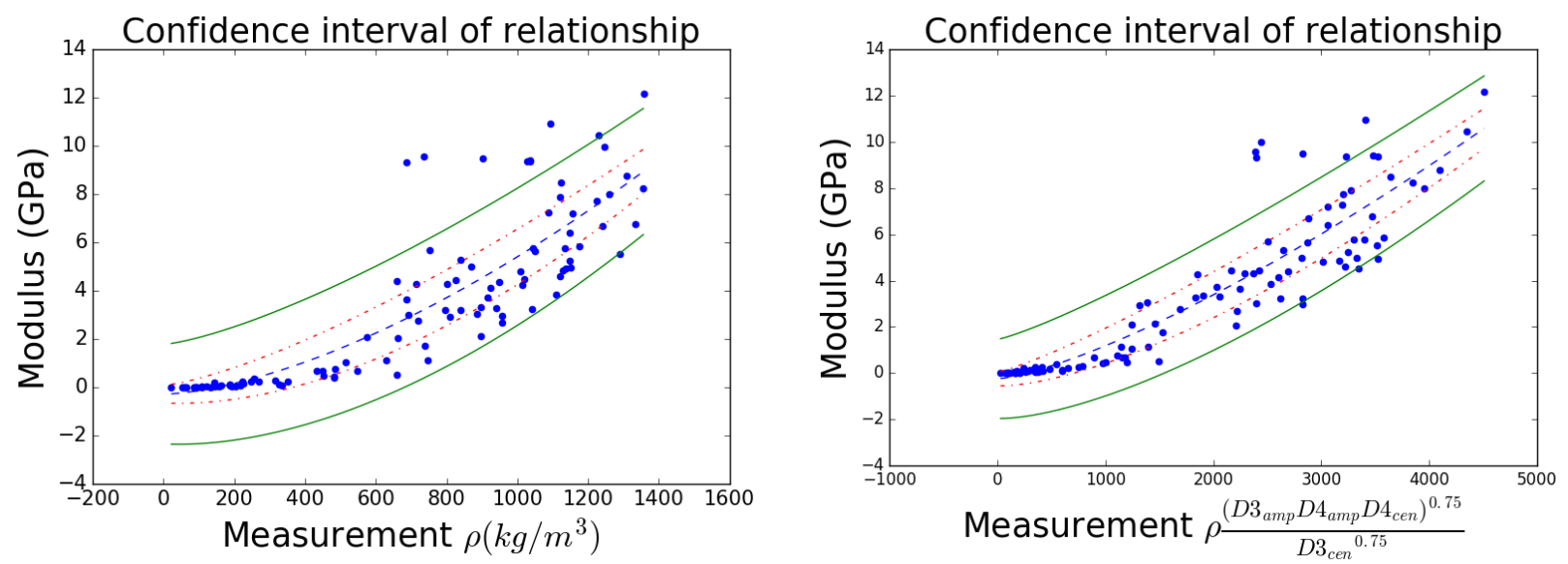

Figure 3.7: The dashed line is the curve fit of the data, the dash dot curves are the $68 \%$ confidence interval, and solid green curves represent the $68 \%$ prediction interval

\subsubsection{Density}

Density is the measurement of how much mass there is in a given area; however, it does not describe what the structure will look like. The infiltrated material is made up decomposed ethylene (the source gas). The carbon fragments formed from the decomposed ethylene can form various kinds of carbon structures. At $700{ }^{\circ} \mathrm{C} \mathrm{CNTs} \mathrm{form} \mathrm{in} \mathrm{a} \mathrm{CVD} \mathrm{process} \mathrm{[35]} \mathrm{[49]} \mathrm{but} \mathrm{at} \mathrm{higher}$ temperatures it can form pyrolitic carbon which is similar to amorphous carbon in how chaotic its 

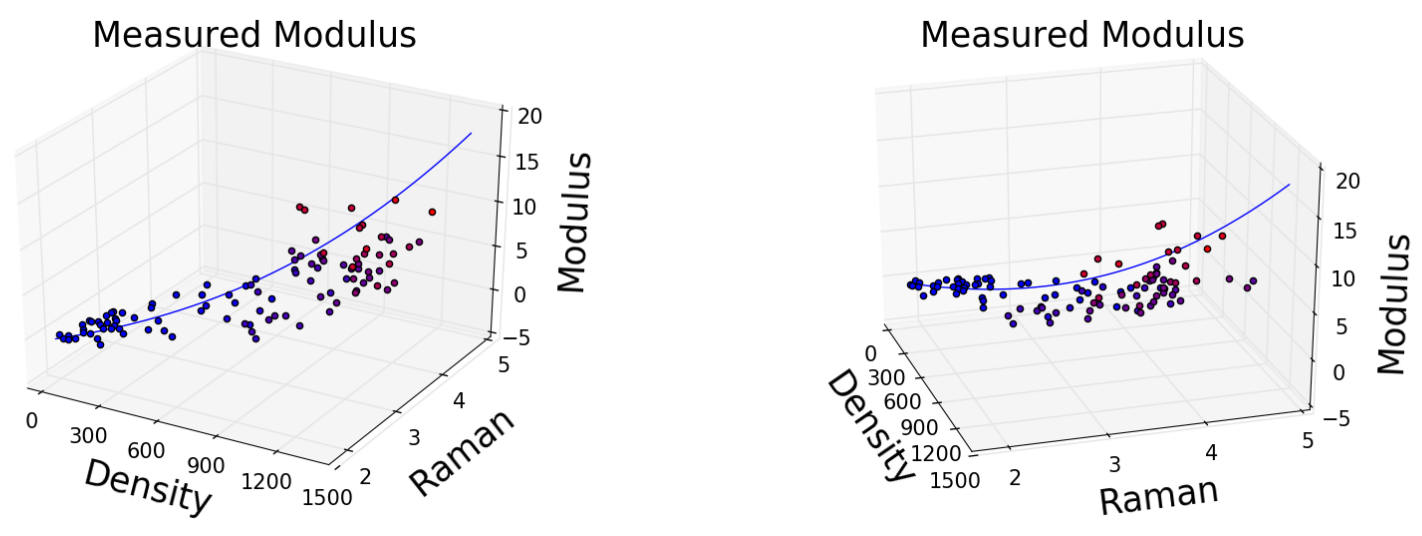

Figure 3.8: Two views of the measured data when plotted by the Raman parameters in equation 3.2, the density, and the bending modulus. The blue line represents the two dimensional fit in figure 3.7 .

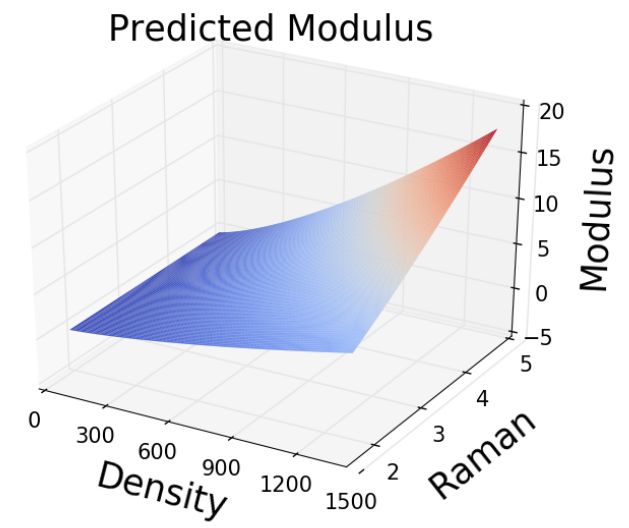

Figure 3.9: A surface plot of the relationship found between Raman spectra, density, and the bending modulus with the color turning red for higher modulus

structure is [50]. Increasing the temperature reduces CNT formation and increases infiltration. The infiltrated carbon joins the CNTs together and forms a denser, stiffer material.

The stiffness of the material significantly increases when CNTs are joined together with infiltrated carbon because the cross-sectional area of material being stressed is increasing (see figure 3.7). Density in CI-CNTs, for the most part, can be correlated with the voids in the structure. In figure 3.10 the relationship between infiltrated CNT width and the average density is mostly linear. The differences are illustrated in high and low infiltrated samples. In Figure 3.11, a low 
infiltrated sample, has a density of $248 \mathrm{~kg} / \mathrm{m}^{3}$ and an infiltrated CNT diameter of $48 \mathrm{~nm}$. Figure 3.2, which is a highly infiltrated sample, has a density of $1009 \mathrm{~kg} / \mathrm{m}^{3}$ and an infiltrated CNT diameter of $125 \mathrm{~nm}$. It is also assumed that the material joining the CNTs has different material properties than the CNTs themselves. Thus, an increase in the infiltrated CNT diameter does not necessarily correlate with an increase in stiffness.

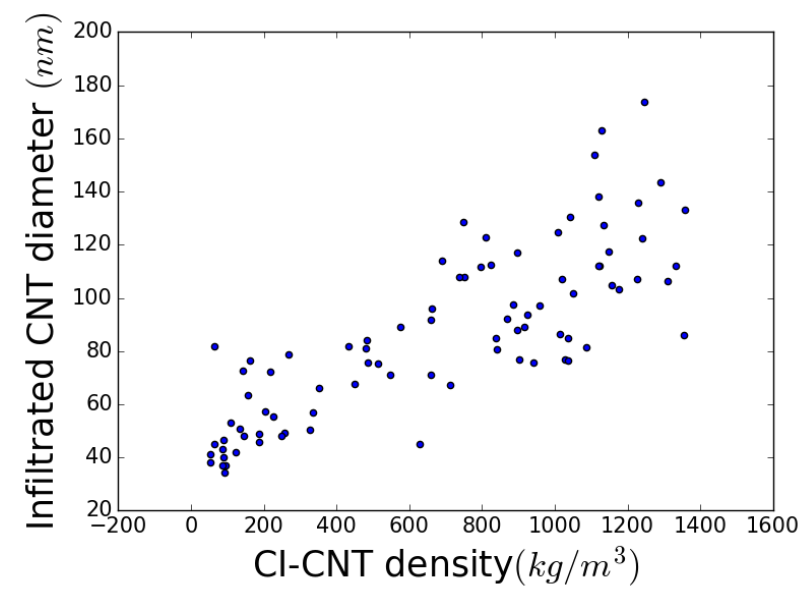

Figure 3.10: The top widths of columns inside CI-CNT beams measured with SEM versus the average density of the beams from the same sample

The Young's Modulus density relationship spreads at higher infiltrations because the infiltration process is not uniform and the type of infiltration changes. Some samples cap quickly, preventing carbon from joining CNTs together throughout the body, and instead build a thick layer on the surface of the beam. Other samples infiltrate more uniformly resulting in different stiffnesses for similar densities. Some samples are infiltrated with a fine carbon structure, while others have what appear to be chunks of infiltrated carbon that started to join to themselves before joining to the CNTs.

\subsection{2 $\mathrm{D}_{3}$ peak}

This peak is associated with amorphous carbon [5] or carbon that is disordered but primarily made of $s p^{2}$ and $s p^{3}$ bonds between carbon [6]. The relationship (eq.3.2) suggests that a $D_{3}$ peak that is further left in the spectrum with a greater amplitude (higher concentration) will mean 


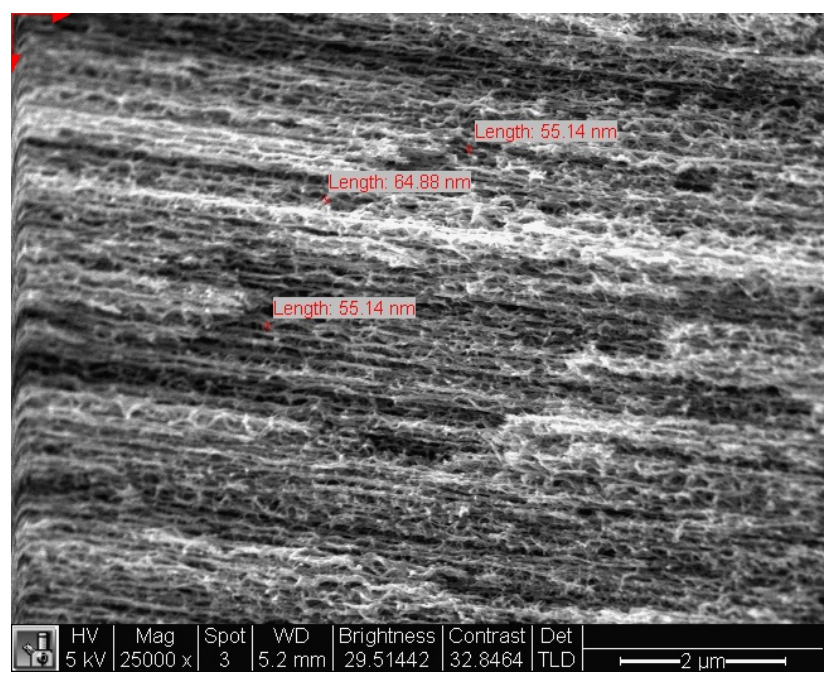

Figure 3.11: SEM image of a part of the cross section of a broken CI-CNT beam infiltrated at $492.4 \mathrm{sccm} \mathrm{H}_{2}$ and $800^{\circ} \mathrm{C}$, for $70 \mathrm{~min}$.

greater stiffness. This can be explained by assuming the $D_{3}$ peak is mostly correlated to the $\mathrm{G}$ peak in amorphous carbon films. Amorphous carbon films are often characterized by $s p^{2}$ and $s p^{3}$ bonding. According to Robertson [6] the $D_{3}$ peak is affected by the $s p^{3}$ concentration (shifting left for higher concentration), and the bond disorder (shifting right for higher clustering). This can be seen in figure 3.12, were the D3 peak is the same as the G peak in the figure. We can assume very little of the $s p^{3}$ bonding comes from the CNTs because they are made of layered graphene tubes. The relationship in eq. 3.2 can then be interpreted to mean that amorphous carbon with higher ratios of $s p^{3}$ bonding, or a greater degree of disorder, results in greater stiffness. The relationship between $s p^{3}$ bonding and stiffness is also seen in some other works with amorphous carbon films such with Dwivedi et. al [51].

\subsubsection{D $_{4}$ peak}

The $D_{4}$ peak has been associated with many different carbon materials. The peak should be associated with a material where both the $D_{4}$ amplitude (concentration) and wave number increase with stiffness. Of the common explanations for the $D_{4}$ peak in figure 3.4 in [4] [5] [18], heteroatoms or polyenes are likely candidates. Based upon the process we used, the most common type of impurity in the material would likely be oxygen. In our study, some CI-CNT beams were sent to MidWest Labs in the USA for CNT analysis, and we found Carbon and Hydrogen made 


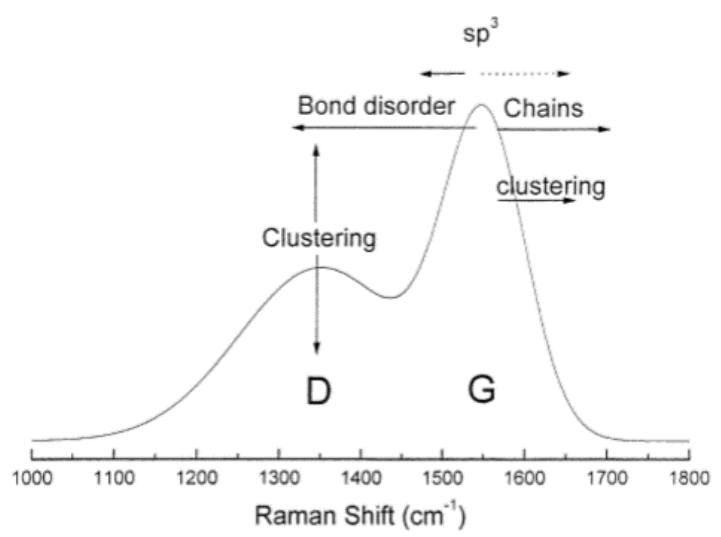

Figure 3.12: Example amorphous carbon Raman spectra from [6] illustrating the affects of bonding on the Raman spectrum

up at least $99.98 \%$ of the samples. Though unlikely, the remaining percentage of unknown atoms could have played a small role in the material stiffness as heteroatoms ( [18] [52]). However, polyenes have a better physical explanation that fits the behavior of the $D_{4}$ peak [17].

In the study done by Kupka et. al [17], the bond length of all-trans carbon molecular chains varies from bond to bond, with the shortest bonds on the ends and the longest bonds in the middle. The shorter the bond length the higher the shift in the Raman spectra [43]. From [17], it can be seen that Raman Spectra of the shorter all-trans carbon chains would have more intense peaks centered at higher wavelength numbers. The shortest chains would have $D_{4}$ peaks around $1200 \mathrm{~cm}^{-1}$ if they exist. Equation 3.2 suggests that stiffer samples have shorter polyenes because stiffer samples have higher $D_{4}$ peak intensities and are centered at higher wave numbers.

Shorter polymer chains causing greater stiffness may seem counter intuitive, but if polyenes are precursors to amorphous carbon then shorter chains seem to be more adept at forming a chaotic structure than long carbon chains. If the polyenes are the results of deformitites or damage to the CNTs from oxidation as in the study by [4], then smaller polyenes would suggest less damage. The first explanation has some support from [7] who suggested that $s p^{2}$ sites in amorphous carbon prefer olefinic ordering. This is also supported by [6] who found that amorphous carbon is more likely to form polyene like structures as $s p^{2}$ sites increase. Having shorter polyenes would reduce the $s p^{2}$ sites, which matches the relationship found in the $D_{3}$ section where higher ratios of $s p^{3}$ to $s p^{2}$ resulted in stiffer materials. 


\subsubsection{Raman parameters and growing conditions}

The randomization used in this study, or the design of experiments method allows correlations to be made between the input parameters and the output D3 and D4 peaks. Using a one-way anova table various relationships were explored. The significant combinations are shown in bold in table 3.3. The $\mathrm{P}$ parameter is the percent chance of the input having no effect. The $\mathrm{F}$ value finds the variance between groups with a minimum variance based upon variance within a group and a $\mathrm{P}$ value less than 0.05 . The parameter D4 represents $D 4_{c e n} D 4_{a m p}$, the D3 parameter represents $\frac{D 3_{a m p}}{D 3_{c e n}}$, and the Raman parameter represents $\frac{D 3_{a m p} D 4_{c e n} D 4_{a m p}}{D 3_{c e n}}$.

Table 3.3: Anova table results

\begin{tabular}{|c|c|c|c|}
\hline Input/Output & F & F crit & P-value \\
\hline Time/D4 & $\mathbf{2 . 9 5 0 3 8 4}$ & $\mathbf{2 . 6 9 2 8 4 1}$ & $\mathbf{. 0 3 6 2 0 4}$ \\
\hline Time/D3 & 0.594734 & 2.692841 & 0.619851 \\
\hline Temp/D4 & $\mathbf{1 8 . 7 8 6 8 6}$ & $\mathbf{2 . 6 9 2 8 4 1}$ & $\mathbf{8 . 5 4 E - 1 0}$ \\
\hline Temp/D3 & $\mathbf{5 5 . 7 0 4 9 3}$ & $\mathbf{2 . 6 9 2 8 4 1}$ & $\mathbf{1 . 7 6 E - 2 1}$ \\
\hline H2/D4 & 0.939889 & 3.083706 & 0.393964 \\
\hline H2/D3 & 1.330347 & 3.083706 & 0.268846 \\
\hline Time/D3*D4 & 2.327147 & 2.692841 & 0.078989 \\
\hline Temp/D3*D4 & $\mathbf{1 5 4 . 9 2 1 6}$ & $\mathbf{2 . 6 9 2 8 4 1}$ & $\mathbf{4 . 9 E - 3 8}$ \\
\hline H2/D3*D4 & 1.162164 & 3.083706 & 0.316837 \\
\hline
\end{tabular}

By plotting the significant relationships in Figure 3.13 it can be seen that increasing the temperature increases the Raman parameters that increase stiffness, while longer infiltration times slowly decrease them. This suggests, from the D3 and D4 sections, that higher temperatures produce a more disordered structure and shorter polyenes. Longer infiltration time likely allows polyenes to grow longer reducing the D4 value.

\subsubsection{SEM Images}

The SEM images taken during this study revealed some trends that help to explain some of the outliers in the model. Of the SEM images taken, capping was found to be one of the most significant factors in variations. 3.14 shows three basic types of images; we categorized each sample imaged as one of these types. In Figure 3.15 samples that are not capped or where the 

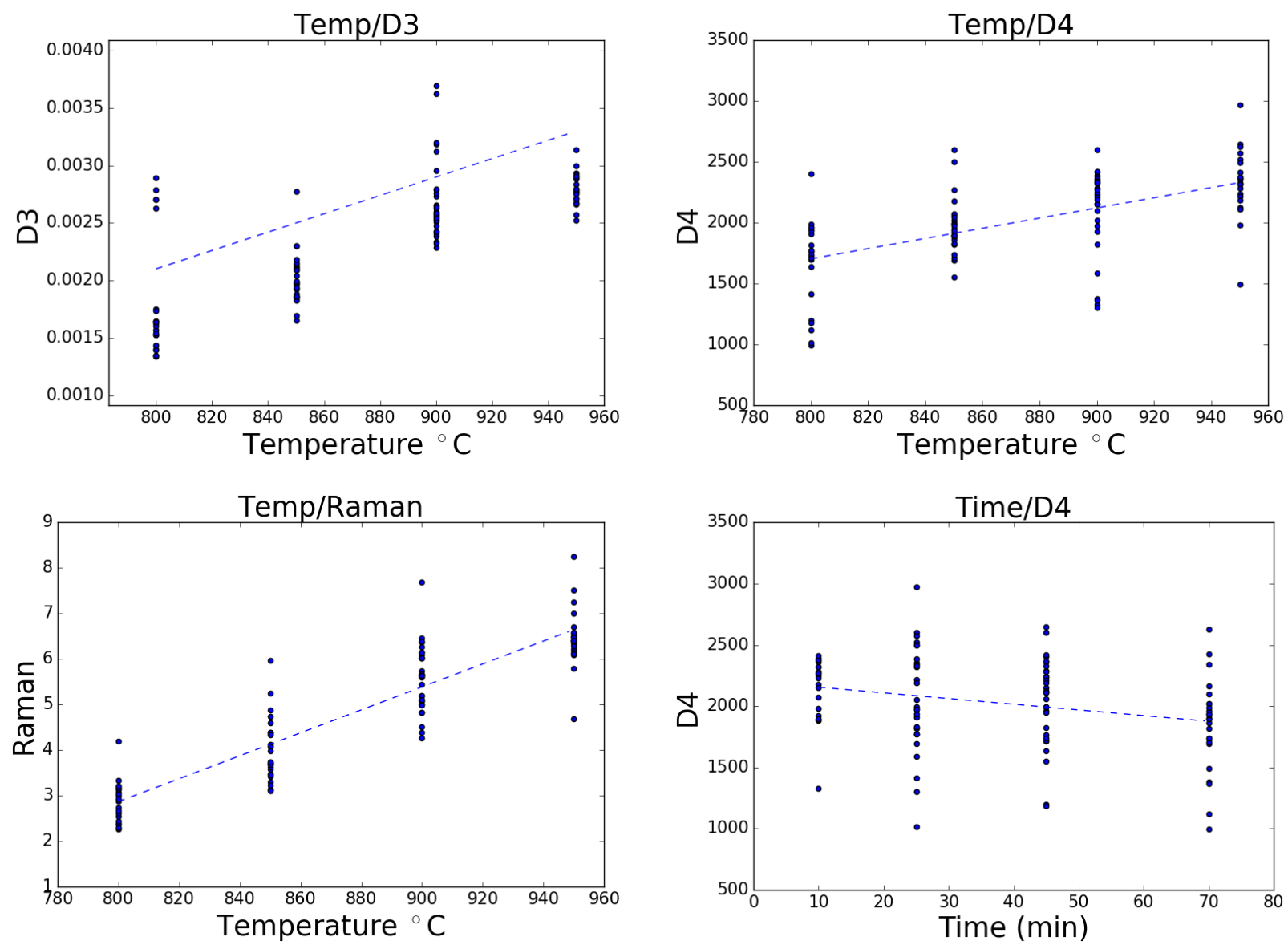

Figure 3.13: Relationships between input and output parameters

cap is smooth, matched well with the model. The thought is that different structures of carbon in the cap can disguise the rest of the structure throughout the CI-CNT beam. As can be seen in figure 3.14 the larger nodules mean a thicker capping layer or more material preventing the Raman spectrum of the interior structure from getting imaged. With the flat types of capping, for example, the interior structure comes close to the surface in some areas whereas the medium sized nodules begin to form almost a thick bush above the interior structure. This causes a trend of ever increasing inaccuracy. Up until nodules become bigger than the columns within the structure, capped samples generally follow the model. Stiffer samples stopped following the model when they were capped and tended to have nodules that were larger than the carbon columns within the structure. These outliers can be brought closer to the model by reducing the Raman power, but the rest of the model spreads more as it approaches the model for density. Samples that were completely capped with 


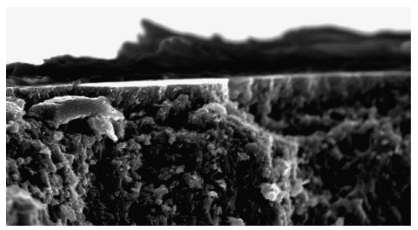

Flat/No Nodule

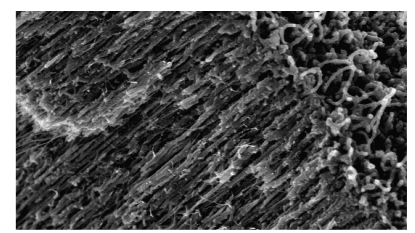

Medium Nodule

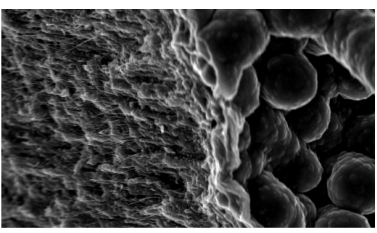

Large Nodule

Figure 3.14: Types of capping identified with SEM

large merged nodules were spread significantly and all but one of the recorded images was over the estimated stiffness.

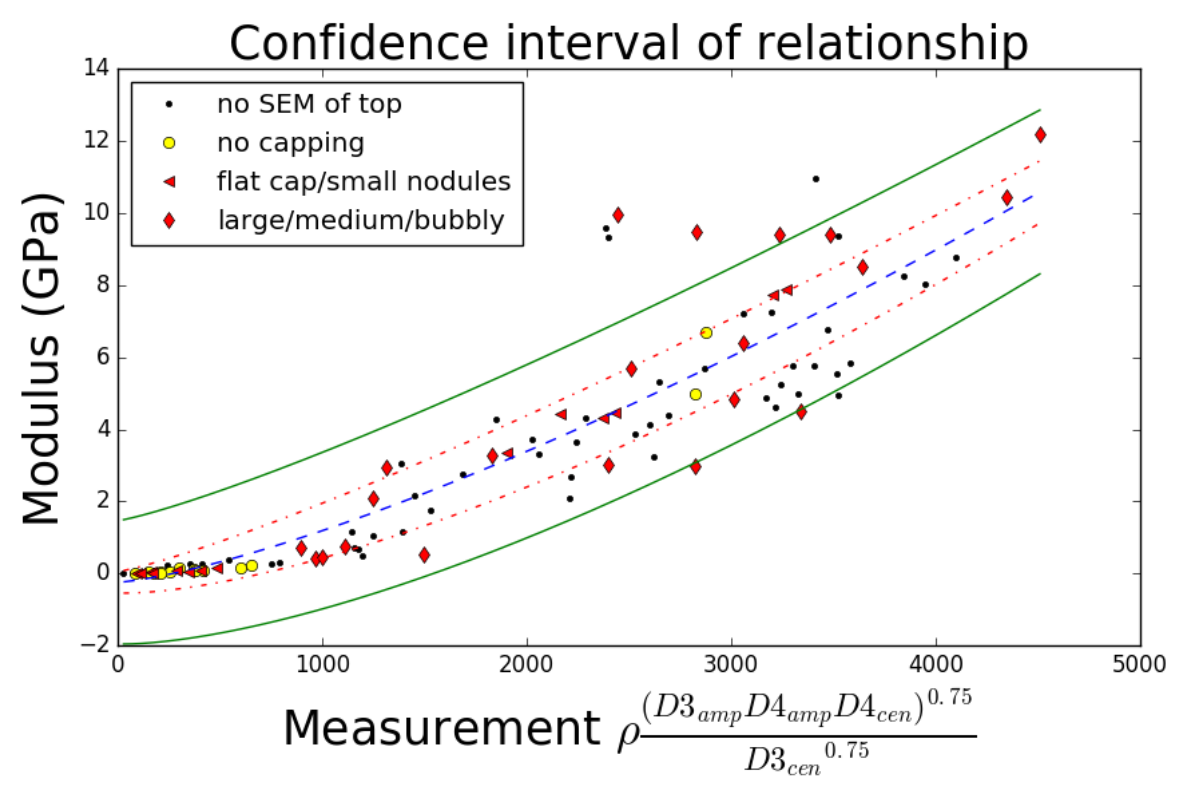

Figure 3.15: Model with SEM capped samples identified

Samples with a thick cap suggest geometry may also play a role in the model underestimating the Young's Modulus of some samples. Equations 3.3 through 3.10 show how the force deflection equation for a simply supported beam can be used to estimate the young's modulus of the capped layer if the total stiffness is known and the internal young's modulus can be estimated. The equations are based upon the dimensions given of the cross-section of a capped CI-CNT beam seen in Figure 3.16. $I_{1}$ and $I_{2}$ are the second moments of area for the middle of the CI-CNT beam and the capped section of the beam respectively with the CNTs in the direction of the width, w. 
The variable $\delta$ represents the displacement, $F$ is the point force exerted at the middle of the beams length, $E$ is the bending modulus, and $L$ is the length of the beam. From equation 3.10 a film with $65 \mathrm{GPa}$ in sample $31(\mathrm{~h}=211 \mu \mathrm{m}, \mathrm{w}=250 \mu \mathrm{m}, \mathrm{t}=2.1 \mu \mathrm{m})$ would make up the difference between the estimated and actual stiffness of sample 31. Films with stiffnesses in this range and higher have been found using plasma enhanced chemical vapor deposition (PECVD) methods which can create carbon ions with more directed energy [53].

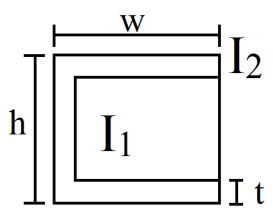

Figure 3.16: Example of how a sample might be capped

$$
\begin{gathered}
F=-\frac{\delta 48 E I}{L^{3}} \\
F_{1}+F_{2}=F_{\text {total }} \text { and } \delta_{1}=\delta_{2}=\delta_{\text {total }} \\
-\frac{\delta 48 E_{1} I_{1}}{L^{3}}-\frac{\delta 48 E_{2} I_{2}}{L^{3}}=-\frac{\delta 48 E_{\text {total }} I_{\text {total }}}{L^{3}} \\
E_{1} I_{1}+E_{2} I_{2}=E_{\text {total }} I_{\text {total }} \\
I_{1}=\frac{(h-2 t)^{3}(w-t)}{12} \\
I_{2}=\frac{h^{3} w}{12}-I_{1} \\
E_{2}=\frac{E_{\text {total }} I_{\text {total }}-E_{1} I_{1}}{I_{2}}
\end{gathered}
$$

In this study samples grown at $800{ }^{\circ} \mathrm{C}$ tended to have a very low stiffness and samples grown at $950{ }^{\circ} \mathrm{C}$ tended to cap. By removing samples from these two extreme combinations samples that may have been capped but did not have SEM imaging done might be removed. The strong concentration of samples with low stiffnesses can also be reduced this way. The resulting 
model can be seen in figure 3.17. While the power on the Raman properties is reduced, they are still important in this relationship. Overall the fit of the model improves to a variance of $1.03 \mathrm{GPa}$.

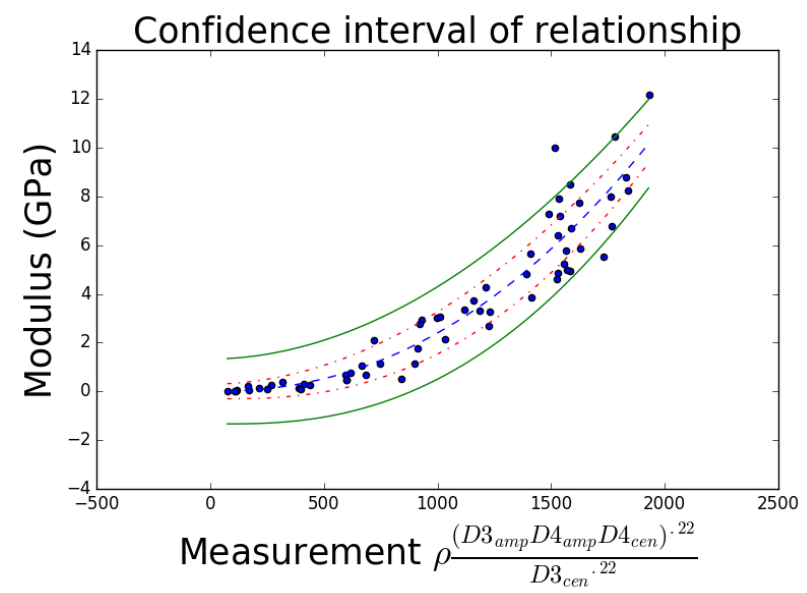

Figure 3.17: Model with samples infiltrated at $950{ }^{\circ} \mathrm{C}$ and $800{ }^{\circ} \mathrm{C}$ removed

\subsubsection{Bending Modulus}

This study tested several CI-CNT beams from each sample and used the average modulus in predicting the model. Some samples had large unexplained variations in bending modulus, as much as $2.46 \mathrm{GPa}$. The range in each sample can be seen in figure 3.18. In the model, the largest variation is found in samples with a bending Modulus of 1.5 GPa or more. The cause for these variations is not known at this time as most beams from a single batch look identical to the naked eye. Since Raman spectra was done on only one beam per sample, under the assumption that they would all be similar, the method of Raman spectra collection may account for some of the outliers. Future work could involve taking SEM pictures of every beam from several samples and then testing them to see the difference.

\subsection{Conclusion}

The stiffness of CI-CNT beams depends upon the type of carbon it is infiltrated with and how much carbon infiltrates the CNT forest. Knowing how much a sample is infiltrated does 


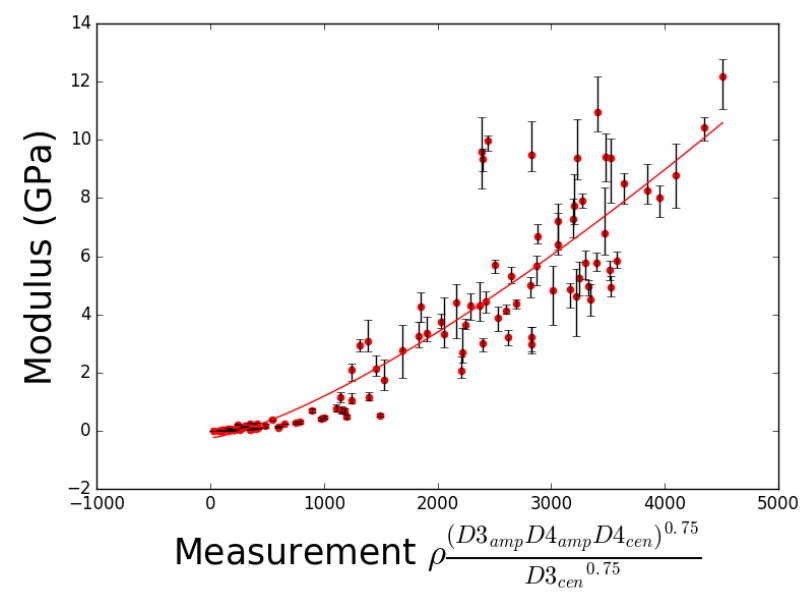

Figure 3.18: Original model but with range of moduli in each sample point labeled

not provide enough information to predict the stiffness of the material. Raman spectroscopy can significantly improve the prediction of CI-CNT Young's Modulus in the direction described in the study. Knowing that such a relationship exists allows for checks to be created when making MEMS devices that need to have stiffnesses in a certain range to work as desired. If CI-CNT materials are not consistently the same stiffness then a method derived from this work may also be used to filter materials. CNTs themselves are frequently grown and then filtered because of difficulty in producing pure batches of a certain kind of CNT.

Raman Spectroscopy results may be less accurate if there are surface defects, and the results might be improved if the top layer is penetrated. It should be checked to see if surface preparation affects CI-CNT beam stiffness before considering it a non-destructive test. As seen in Figure 3.16 and in equation 3.10 a capped layer can be very thin (thus susceptible to damage) but have a large impact on the total stiffness. Removing the capped layer with surface prep thus could change the bending modulus of the beam.

The stiffness of CI-CNT beams grown in a tube furnace can be estimated, with a $68 \%$ prediction interval of $4.9 \mathrm{GPa}$ with the non-destructive methods used in this study. This may seem like a large range but it still allows the $12 \mathrm{GPa}$ range in this study to be subdivided into different groups. This range was also achieved by only changing parameters related to infiltration. As mentioned in the introduction section other parameters in the process of growing CI-CNT beams can also affect the stiffness. If the CNTs change significantly in shape and size the relationship 
may be different then depicted in this study. However it is expected that density and the same Raman properties as in this study will be in a model where CNTs are also changed. 


\section{CHAPTER 4. SUMMARY AND FUTURE WORK}

In this chapter the results of the study are summarized. Future work that can be done for improving the study as well as contributions are also discussed.

\subsection{Results Summary}

The key result from the study is the relationship between Raman spectroscopy, density, and bending modulus (see equation 4.1). This relationship allows the bending modulus of CI-CNT structures to be estimated by just weighing the sample, measuring its geometry, and taking an optical scan with Raman. This non-destructive method preserves the potentially expensive sample for further use.

Elastic modulus $(\mathrm{GPa})=1.3351 \times 10^{-4}\left(\rho\left(\frac{D 3_{a m p} D 4_{a m p} D 4_{c e n}}{D 3_{c e n}}\right)^{.75}\right)^{1.3435}-0.2485$

In this study a large variety of CI-CNT beams were tested. As seen in table 4.1 a range of stiffnesses were tested, allowing for a large range of CI-CNTs to be predicted via equation 4.1. Some errors, as mentioned in chapter 3 , can be attributed to inconsistencies in the samples. In Table 4.1 illustrates just how much variation can be in one sample. Since density and Raman were measured once for the entire sample all of the differences in the CI-CNT beams fail to be captured in the study.

Chapter 2 discussed the different Raman peaks used in the study. The position ranges of each peak in the study, and their average positions are given in table 4.2. While several peaks can have widely different positions in different CI-CNTs some peaks such as the G peak moved very little. The peak positions were determined using the lmfit optimizer written for Python. 
Table 4.1: Material Properties of CI-CNT beams

\begin{tabular}{|c|c|c|c|}
\hline Property & Maximum sample average & Minimum sample average & $\begin{array}{c}\text { Maximum Range } \\
\text { in a given sample }\end{array}$ \\
\hline Bending Modulus & $12.184 \mathrm{GPa}$ & $0.003 \mathrm{GPa}$ & $2.457 \mathrm{GPa}$ \\
\hline Density & $1357.3 \mathrm{~kg} / \mathrm{m}^{3}$ & $22.0 \mathrm{~kg} / \mathrm{m}^{3}$ & \\
\hline Height & $633 \mu \mathrm{m}$ & $224 \mu \mathrm{m}$ & $140 \mu \mathrm{m}$ \\
\hline Width & $227 \mu \mathrm{m}$ & $180 \mu \mathrm{m}$ & \\
\hline
\end{tabular}

Table 4.2: Peaks in CI-CNT Raman spectra

\begin{tabular}{|c|c|c|c|}
\hline Peak & Maximum position $\mathrm{cm}^{-1}$ & Minimum position $\mathrm{cm}^{-1}$ & Average Position $\mathrm{cm}^{-1}$ \\
\hline $\mathrm{D}_{1}$ & 1333 & 1315 & 1324 \\
\hline $\mathrm{D}_{2}$ & 1672 & 1596 & 1637 \\
\hline $\mathrm{D}_{3}$ & 1535 & 1485 & 1515 \\
\hline $\mathrm{D}_{4}$ & 1188 & 1170 & 1178 \\
\hline $\mathrm{G}$ & 1603 & 1591 & 1600 \\
\hline $\mathrm{D}_{5}$ & 1930 & 1830 & 1873 \\
\hline
\end{tabular}

\subsection{Contributions}

This thesis was part of a larger project aimed at better understanding CI-CNT beams and producing stiffer and stronger CI-CNT beams. My primary contributions were in taking Raman spectra of CI-CNT beams, weighing them, writing code to find the bending modulus of the beams, and analyzing the data to find the relationship in equation 4.1.

This thesis contributes to the knowledge of CI-CNT beams by providing a method for estimating beam stiffness and identifying key properties that make stiffer CI-CNT beams. In chapter 3 , the $\mathrm{D}_{3}$ peak and the $\mathrm{D}_{4}$ peak are related to different carbon structures and explain how certain changes in the structures can increase stiffness of CI-CNT beams.

A smaller, yet important, contribution of this work is the emphasis on charged couple device (CCD) calibrations. Some journal articles use the default calibration settings of commercial spectrometer products, but as in the case of this study, the calibrated spectra may still be incorrect if not checked in some way. 


\subsection{Future Work}

While this study provides a relationship for predicting the bending modulus it does raise a few questions. Would the relationship be improved if the beams could be weighed and scanned individually? How much of an effect does capping of CI-CNT beams really have on the beams? Does the relationship get worse as samples get stiffer?

Some of these questions may be answered with a few different methods in future research. The capping question could be solved by carefully removing the cap of CI-CNT beams before taking a Raman spectrum. The cap may be removed by a diluted acidic solution or even some form of laser in a vacuum environment. The process has the potential to be time consuming but would illustrate the affects of having a capped sample by comparing it to an identical sample without a cap.

Weighing individual beams with standard equipment will likely continue to be difficult. Creative methods such as weighing them in a vacuum or vibrating a small piezoelectric beam with a CI-CNT sample on the end may be one way of measuring such small beams. Taking Raman spectra of individual beams is very possible; it just takes time. The spectra also need to be taken before the beams are broken as beam fragments are usually difficult to gather after breaking.

Overall, this study shows that CI-CNT properties such as the bending modulus can be determined with non-destructive methods. 


\section{REFERENCES}

[1] N. B. Colthup, L. H. Daly, and S. E. Wiberly, Introduction to infrared and Raman spectroscopy, 2nd ed. Academic Press, 1975. vii, 2, 4, 5, 6, 22

[2] J. R. Ferraro, K. Nakamoto, and C. W. Brown, Introductory Raman Spectroscopy, 2nd ed. 525 B Street 1900, San Diego, California 92101-4495, USA: Academic Press, 2003. vii, 4, 5,6

[3] M. Szybowicz, A. B. Nowicka, and A. Dychalska, "Chapter 1 - characterization of carbon nanomaterials by raman spectroscopy," in Characterization of Nanomaterials, ser. Micro and Nano Technologies, S. M. Bhagyaraj, O. S. Oluwafemi, N. Kalarikkal, and S. Thomas, Eds. Woodhead Publishing, 2018, pp. 1 - 36. [Online]. Available: http://www.sciencedirect.com/science/article/pii/B9780081019733000018 vii, 7, 8

[4] S. A. Chernyak, A. S. Ivanov, K. I. Maslakov, A. V. Egorov, Z. Shen, S. S. Savilov, and V. V. Lunin, "Oxidation, defunctionalization and catalyst life cycle of carbon nanotubes: a raman spectroscopy view," Phys. Chem. Chem. Phys., vol. 19, pp. 2276-2285, 2017. [Online]. Available: http://dx.doi.org/10.1039/C6CP04657F vii, 7, 8, 13, 14, 22, 23, 31, 32

[5] A. Sadezky, H. Muckenhuber, H. Grothe, R. Niessner, and U. Pschl, "Raman microspectroscopy of soot and related carbonaceous materials: Spectral analysis and structural information," Carbon, vol. 43, no. 8, pp. 1731 - 1742, 2005. [Online]. Available: http://www.sciencedirect.com/science/article/pii/S0008622305001247 vii, 9, 10, 11, 12, 13, $14,15,23,24,30,31$

[6] J. Robertson, "Diamond-like amorphous carbon," Materials Science and Engineering: R: Reports, vol. 37, no. 4-6, pp. 129-281, 2002. vii, viii, 10, 12, 14, 18, 22, 24, 30, 31, 32

[7] K. Jones, B. D. Jensen, and A. Bowden, "Fabrication and testing of planar stent mesh designs using carbon-infiltrated carbon nanotubes," Journal of Nanotechnology in Engineering and Medicine, vol. 4, no. 2, Oct. 2013. 1, 17, 18, 32

[8] S. Rodil, R. Olivares, H. Arzate, and S. Muhl, "Properties of carbon films and their biocompatibility using in-vitro tests," Diamond and related materials, vol. 12, pp. 931-937, 2003. 1

[9] X. Hou, Y. He, and B. T. Jones, "Recent advances in portable x-ray flourescence spectrometry," Applied Spectroscopy Reviews, vol. 39, no. 1, pp. 1-25, 2004. 1

[10] Q. Zhao, Y. Liu, L. Wang, H. Yang, and D. Cao, "Design method for piezoelectric cantilever beam structure under low frequency condition," International Journal of Pavement Research and Technology, vol. 11, pp. 153-159, 2018. 2 
[11] "Spectral database for organic compounds SDBS (National Institute of Advanced Industrial Science and Technology)," https://sdbs.db.aist.go.jp, accessed: 2018-09-28. 2, 22

[12] M. Castriota, E. Cazzanelli, L. Caputi, A. Cupolillo, C. Giallombardo, L. Papagno, and G. Mariotto, "Investigations on raman bands from carbon linear chains in multiwalled carbon nanotubes," Diamond and Related Materials, vol. 17, no. 7, pp. 1716 - 1723, 2008, proceedings of Diamond 2007, the 18th European Conference on Diamond, Diamond-Like Materials, Carbon Nanotubes, Nitrides and Silicon Carbide. [Online]. Available: http://www.sciencedirect.com/science/article/pii/S0925963508000745 7, 14

[13] M. Dresslhaus, G. Dresselhaus, R. Saito, and A. Jorio, "Raman spectroscopy of cabron nanotubes," Physics Reports, vol. 409, pp. 47-99, 2005. 7, 14, 16

[14] R. J. Nemanich and S. A. Solin, "First- and second-order raman scattering from finite-size crystals of graphite," Phys. Rev. B, vol. 20, pp. 392-401, Jul 1979. [Online]. Available: https://link.aps.org/doi/10.1103/PhysRevB.20.392 9, 16, 23

[15] A. C. Ferrari and J. Robertson, "Raman spectroscopy of amorphous nanostructured, diamondlike carbon, and nanodiamond," The Royal Society, vol. 362, pp. 2477-2512, 2004. 10, 14

[16] P. J. Larkin, "Chapter 6 - ir and raman spectrastructure correlations: Characteristic group frequencies," in Infrared and Raman Spectroscopy (Second Edition), second edition ed., P. J. Larkin, Ed. Elsevier, 2018, pp. 85 - 134. [Online]. Available: http://www.sciencedirect.com/science/article/pii/B9780128041628000069 11

[17] T. Kupka, A. Buczek, M. A. Broda, M. Stachw, and P. Tarnowski, "Dft studies on the structural and vibrational properties of polyenes," Journal of Molecular Modeling, vol. 22, 2016. 11,32

[18] M. W. Smith, I. Dallmeyer, T. J. Johnson, C. S. Brauer, J.-S. McEwen, J. F. Espinal, and M. Garcia-Perez, "Structural analysis of char by raman spectroscopy: Improving band assignments through computational calculations from first principles," Carbon, vol. 100, pp. 678 - 692, 2016. [Online]. Available: http://www.sciencedirect.com/science/article/pii/ S0008622316300318 13, 31, 32

[19] M. Veres, M. Füle, S. Tóth, M. Koós, and I. Pócsik, "Surface enhanced raman scattering (SERS) investigation of amorphous carbon," Diamond and Related Materials, vol. 13, no. 4, pp. 1412 - 1415, 2004, 14th European Conference on Diamond, DiamondLike Materials, Carbon Nanotubes, Nitrides and Silicon Carbide. [Online]. Available: http://www.sciencedirect.com/science/article/pii/S0925963504000810 13

[20] M. Rybachuk and J. Bell, "Electronic states of trans-polyacetylene, poly(p-phenylene vinylene) and sp-hybridised carbon species in amorphous hydrogenated carbon probed by resonant raman scattering," Carbon, vol. 47, no. 10, pp. 2481 - 2490, 2009. [Online]. Available: http://www.sciencedirect.com/science/article/pii/S000862230900284X 13

[21] S. Silva, J. Carey, R. Khan, E. Gerstner, and J. Anguita, "Chapter 9 - amorphous carbon thin films," in Handbook of Thin Films, H. S. Nalwa, Ed. Burlington: Academic Press, 
2002, pp. 403 - 506. [Online]. Available: http://www.sciencedirect.com/science/article/pii/ B978012512908450059X 13, 14

[22] C. Casiraghi, A. C. Ferrari, and J. Robertson, "Raman spectroscopy of hydrogenated amorphous carbons," Physical Review B, vol. 72, p. 085401, 2005. 14

[23] B. B. He, Two-dimensional x-ray diffraction. 111 River Street, Hoboken, NJ 07030, USA: John Wiley \& Sons, Inc, 2018. 15

[24] J.-L. Sauvajol, K. Chesnel, E. Anglaret, R. Almairac, R. Aznar, P. Boutrouille, and B. Hennion, "Inelastic neutron scattering investigation of quenched $\csc _{6} 0$ phases," Solid State Communications, vol. 108, no. 10, pp. 781-785, 1998. 15

[25] F. Hibbert, E. Geissler, J. L. Hodeau, E. Lelévre-berna, and J.-R. Regnard, Neutron and X-ray Spectroscopy. P.O. Box 17, 3300 AA Dordrecht, The Netherlands: Springer, 2006. 15

[26] V. Mlynárik, "Introduction to nuclear magnetic resonance," Analytical Biochemistry, vol. 529, pp. 4 - 9, 2017, introduction to in vivo Magnetic Resonance Spectroscopy (MRS): A method to non-invasively study metabolism. [Online]. Available: http: //www.sciencedirect.com/science/article/pii/S0003269716300719 16

[27] D. McCulloch, D. McKenzie, and C. Goringe, "Ab initio simulations of the structure of amorphous carbon,” Physical Review B, vol. 61, no. 3, pp. 2349-2355, 2000. 16

[28] D. Caschera, F. Federici, S. Kaciulis, L. Pandolfi, A. Cusma, and G. Padeletti, "Depostion of ti-containing diamond-like carbon (dlc) films by pecvd technique," Materials Science and Engineering C, vol. 27, pp. 1328-1330, 2007. 17

[29] E. J. Dr., "Electronic structure and chemical reactivity of carbon nanotubes: A chemist's view," ChemPhysChem, vol. 5, 2004. 17

[30] K. Moulton, N. B. Morrill, A. M. Konneker, B. D. Jensen, R. R. Vanfleet, D. D. Allred, and R. C. Davis, "Effect of iron catalyst thickness on vertically aligned carbon nanotube forest straightness for cnt-mems," Journal of Micromechanics and Microengineering, vol. 22, 2012. 17

[31] K. A. Stevens, C. D. Esplin, T. M. Davis, D. J. Butterfield, P. S. Ng, A. E. Bowden, B. D. Jensen, and B. D. Iverson, "Superhydrophobic, carbon-infiltrated carbon nanotubes on si and 3161 stainless steel with tunable geometry," Applied Phisics Letters, vol. 112, p. 211602 , 2018. 17

[32] W. C. Fazio, J. M. Lund, T. S. Wood, B. D. Jensen, R. C. Davis, and R. R. Vanfleet, "Material properties of carbon-infiltrated carbon nanotube-templated structures for microfabrication of compliant mechanisms," in ASME 2011 International Mechanical Engineering Congress and Exposition, vol. 11: Nano and Micro Materials, Devices and Systems; Microsystems Integration. Denver, Colorado, USA: ASME, November 2011, pp. 481-490. 17

[33] H. B. Hanna, W. C. Fazio, J. D. Tanner, J. M. Lund, T. S. Wood, R. C. Davis, R. R. Vanfleet, and B. D. Jensen, "Mechanical property measurement of carbon infiltrated carbon nanotube 
structures from compliant micromechanisms," Journal of Microelectromechanical Systems, vol. 23, no. 6, pp. 1330-1339, Dec. 2014. 17, 25

[34] L. L. Howell, Compliant Mechanisms. 605 Third Avenue, New York, NY: John Wiley \& Sons, Inc, 2001. 17

[35] A. Shaikjee and N. J. Coville, "The role of the hydrocarbon source on the growth of carbon materials," Carbon, vol. 50, no. 10, pp. 3376 - 3398, 2012. [Online]. Available: http://www.sciencedirect.com/science/article/pii/S0008622312002722 18, 28

[36] Y. Homma, Y. Kobayashi, T. Ogino, D. Takagi, R. Ito, Y. Jung, and P. Ajayan, "Role of transition metal catalysts in single-walled carbon nanotube growth in chemical vapor deposition," Journal of Physical Chemistry B, vol. 107, pp. 12 161-12 164, 2003. 18

[37] S. Doorn, D. Heller, P. Barone, M. Usrey, and M. Strano, "Resonant raman excitation profiles of individually dispersed single walled carbon nanotubes in solution," Applied Physics A, vol. 78, no. 8, pp. 1147-1155, 2004. 21

[38] M. Ess, E. Kireeva, R. Niessner, F.-X. Ouf, and N. Ivleva, "In situ raman microspectroscopic analysis of soot samples with different organic carbon content: Structural changes during heating," Carbon, vol. 105, pp. 572-585, 2016. 22

[39] S. ai and Y. Ozaki, Eds., Raman Infrared, and Near-Infrared Chemical Imaging. John Wiley \& Sons, Inc., 2010. 22

[40] "Handbook of minerals raman spectra (Laboratoire de gologie de Lyon ENS de Lyon France)," http://www.geologie-lyon.fr/Raman/index.php, 2000-2018. 22

[41] "Vibrational properties of polymer and quenched $\mathrm{Cs}_{60}$ phases," J. Chim. Phys., vol. 95, pp. 1441-1444, 1998. 22

[42] I. S. McLean, Electronic Imaging in Astronomy Detectors and Instrumentation. John Wiley \& Sons Ltd in association with Praxis Publishing Ltd, 1997. 23

[43] Z. H. Ni, T. Yu, Y. H. Lu, Y. Y. Wang, Y. P. Feng, and Z. X. Shen, "Uniaxial strain on graphene: Raman spectroscopy study and band-gap opening," ACS Nano, vol. 2, no. 11, pp. 2301-2305, 2008, pMID: 19206396. [Online]. Available: https://doi.org/10.1021/nn800459e 24,32

[44] E. Mohagheghpour, M. Rajabi, R. Gholamipour, M. Larijani, and S. Sheibani, "Correlation study of structural, optical and electrical properties of amorphous carbon thin films prepared by ion beam sputtering deposition technique," Applied Surface Science, vol. 360, pp. 52-58, 2016. 24

[45] A. Dychalska, P. Popielarski, W. Franków, K. Fabisiak, K. Paprocki, and M. Szybowicz, "Study of cvd diamond layers with amorphous carbon admixture by raman scattering spectroscopy," Materials Science-Poland, vol. 33, no. 4, pp. 799-805, 2015. 24

[46] ASTM Standard D790, "Standard Test Methods for Flexural Properties of Unreinforced and Reinforced Plastics and Electrical Insulating Materials," in Annual Book of ASTM Standards. West Conshohocken, PA: ASTM International, 2014. 25 
[47] R. G. Budynas and J. K. Nisbett, Mechanical Engineering Design, 9th ed. 1221 Avenue of the Americas, New York, NY 10020: McGraw-Hill, 2011. 25

[48] M. B. Priestley, Spectral Analysis and Time Series, Z. W. Birnbaum and E. Lukacs, Eds. Academic Press, 1981, vol. 1. 27

[49] R. Andrews, D. Jacques, D. Qian, and E. Dickey, "Purification and structural annealing of multiwalled carbon nanotubes at graphitization temperatures," Carbon, vol. 39, no. 11, pp. 1681 - 1687, 2001. [Online]. Available: http://www.sciencedirect.com/science/article/pii/ S0008622300003018 28

[50] B. K. Kwieciska and S. Pusz, "Pyrolytic carbon definition, classification and occurrence," International Journal of Coal Geology, vol. 163, pp. 1 - 7, 2016. [Online]. Available: http://www.sciencedirect.com/science/article/pii/S016651621630307X 29

[51] N. Dwivedi, S. Kumar, H. Malik, Govind, C. Rauthan, and O. Panwar, "Correlation of sp3 and sp2 fraction of carbon with electrical, optical and nano-mechanical properties of argondiluted diamond-like carbon films," Applied Surface Science, vol. 257, pp. 6804-6810, 2011. 31

[52] R. Cammack, T. Atwood, P. Campbell, H. Parish, A. Smith, F. Vella, and J. Stirling, Eds., Oxford Dictionary of Biochemistry and Molecular Biology (2 ed.). Oxford Univrsity Press, 2006. 32

[53] F. Piazza, D. Grambole, D. Schneider, C. Casiraghi, A. Ferrari, and J. Robertson, "Protective diamond-like carbon coatings for future optical storage disks," Diamond \& Related Materials, vol. 14, pp. 994-999, 2005. 36 


\section{APPENDIX A. RAMAN SPECTRUM FITTING}

The following is the python script for fitting the Raman spectra.

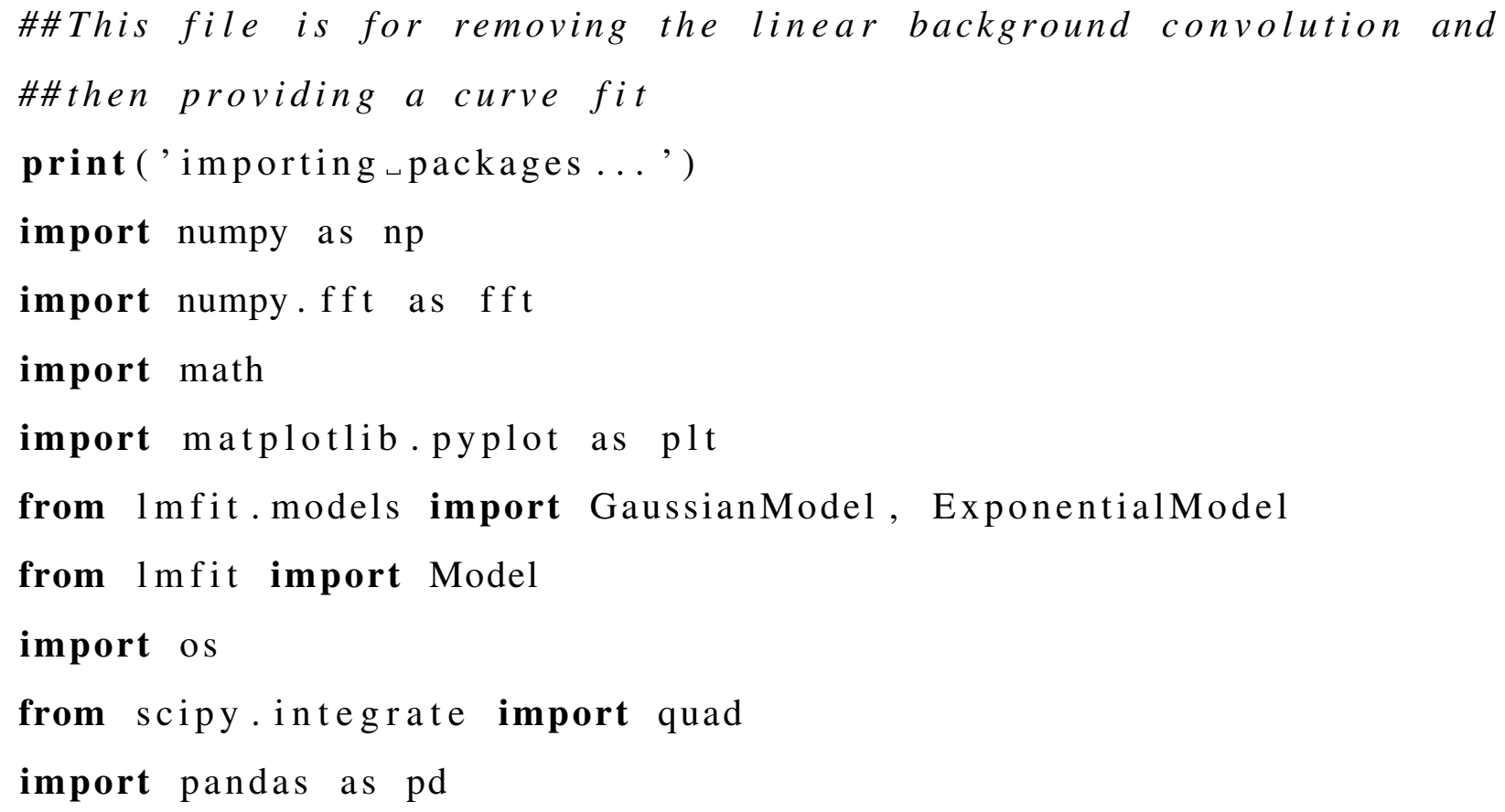


from functions 2 import Triangle

from functions 2 import Gauss_func

def LorenztianModel (x, amp, cen, Q, F) :

$$
\begin{aligned}
& \text { top }=(1+2 *(\mathrm{x}-\text { cen }) /(\mathrm{Q} * \mathrm{~F})) * * 2 \\
& \text { bot }=1+(2 *(\mathrm{x}-\text { cen }) / \mathrm{F}) * * 2 \\
& \text { return amp } * \text { top } / \text { bot }
\end{aligned}
$$

def LinearModel(x, slope, intercept):

return $x *$ slope+intercept

\#\#LOAD DATA\#\#

print ('loading $\sqcup$ data ...')

os.chdir ('Data $\_-\_$Copy')

items=os. 1 istdir $\left({ }^{\prime}, '\right)$

fname $e_{-}$ist $=[]$

\#List of files to be uploaded

fil e=open (" ou tput . csv", , "w")

\#Create file to record ratios

file . write ("Growth $₫$ \#, L1_slope, L1_intercept , G2_center ,")

file . write ("G2_height, D1_amp, D1_cen, D2_amp,")

fil e . write ("D2_cen ,D3_amp, D3_cen ,D4_amp, D4_cen, G_amp, G_cen ,")

file .write ("G2_area, D1_area,D2_area, D3_area, D4_area, G_area, Laser $, \backslash \mathrm{n} ")$

$\mathrm{i}=0$

$\mathrm{k}=0$

sample_number $=[]$ 
Al1_corrections=pd. DataFrame. from_csv ('correction . csv', header $=0$ ) ratio $=$ All_corrections ['Correction $\lrcorner$ Ratio'] thermal=A11_corrections ['Thermal_Correction']

items $2=n p \cdot z \operatorname{zeros}([\mathbf{l e n}($ items $), 3])$. astype ('str')

\#\#Pick only the latest file versions\#\#

for $j$ in range(len(items)):

temp $=\left(\right.$ items $\left.[j] . \operatorname{split}\left({ }^{\prime},{ }^{\prime}\right)[0]\right) \cdot \operatorname{split}\left({ }^{\prime}\left({ }^{\prime}, 1\right)\right.$

if $\operatorname{len}($ temp $)>=2$ :

temp=np . append (temp [0], temp [1]. split (')') [0])

1 oc $=$ np. where $($ items $2==$ temp $[0])$

if $\operatorname{len}(\operatorname{loc}[0])==0$ :

$1 \mathrm{oc}=\mathrm{np} \cdot$ where $($ item $\mathrm{s}==\operatorname{temp}[0][:-1])$

if $\operatorname{len}(\operatorname{loc}[0])==0$ :

$1 \mathrm{oc}=\mathrm{np} \cdot$ where $($ items $2==(\operatorname{temp}[0]+" \sqcup "))$

if $\operatorname{len}(\operatorname{loc}[0])==0$ :

for $\mathrm{n}$ in range(len (temp)):

items $2[\mathrm{k}, \mathrm{n}]=$ temp $[\mathrm{n}]$

$\mathrm{k}=\mathrm{k}+1$

elif len (temp) $>1$ :

if float (items2[1oc[0], 1][0])<float (temp [1]):

items $2[\operatorname{loc}[0], 1]=$ temp [1]

\#\#Re-stitch\#\#

items $=[]$

remove $=0$

for $j$ in range(len(items 2$))$ :

if items $2[\mathrm{j}][0]=={ }^{\prime} 0.0^{\prime}$ :

remove $=$ remove +1

elif items2[j][1]==' $0.0{ }^{\prime}$ : 


$$
\text { items }=n p . \text { append }\left(\text { items },\left(\text { items } 2[j, 0]+{ }^{\prime} \cdot t x t t^{\prime}\right)\right)
$$

else :

$$
\begin{aligned}
& \text { items }=n p . \text { append }\left(\text { items },\left(\text { items } 2[j, 0]+{ }^{\prime}\left({ }^{\prime}+\text { items } 2[j\right.\right.\right. \\
& \left.\left.\left., 1]+{ }^{\prime}\right) \cdot t \times t^{\prime}\right)\right)
\end{aligned}
$$

for $\mathrm{j}$ in range(remove):

items $2=n p \cdot$ delete $($ items $2,-1,0)$

\#\#Remove ones on the NA list\#\#

$\mathrm{j}=-1$

for $k$ in range(len (items)):

$$
\begin{aligned}
& j=j+1 \\
& \text { temp=items }[j] . \operatorname{split}\left({ }^{-},\right. \\
& \text {if } \operatorname{len}(\operatorname{temp})>3 \text { : }
\end{aligned}
$$

$$
\begin{gathered}
\text { if temp }[2]=={ }^{\prime} 10 \mathrm{~min} \text { ' and temp }[3]=={ }^{\prime} 800 \mathrm{C}^{\prime}: \\
\text { items }=\mathrm{np} \text {. delete }(\text { items }, \mathrm{j}) \\
\mathrm{j}=\mathrm{j}-1
\end{gathered}
$$

elif temp $[3]=={ }^{\prime} 950 \mathrm{C}^{\prime}$ and temp $[2]=={ }^{\prime} 70 \mathrm{~min}$ ' and temp $[1] !={ }^{\prime} 95^{\prime}$ :

np.delete (items, j )

elif temp $[1]=={ }^{\prime} 80$ ' and temp $[2]=={ }^{\prime} 70 \mathrm{~min}$ ' and temp

$$
[3]=={ }^{\prime} 800 \mathrm{C}^{\prime} \text { : }
$$$$
\text { np.delete (items, j) }
$$

elif temp $[1]=={ }^{\prime} 80^{\prime}$ and temp $[2]=={ }^{\prime} 25 \mathrm{~min}$ ' and temp

$$
\begin{aligned}
{[3]==} & ' 850 C^{\prime}: \\
& n p \cdot \text { delete }(\text { items }, j)
\end{aligned}
$$

\#\#Pick which files to analyze\#\#

for names in items:

$$
\mathrm{i}=\mathrm{i}+1
$$


if $\quad \mathrm{i}==1000$ :

\#Decides the maximum number of files to analyze

break

if (names.endswith(".txt") and names $!="$ output.txt" and names !="Density . txt") :

if names $[-5]==")$ : :

if names $[-13]=={ }^{\prime} 7$ :

fname $\_$ist $=n p$. append ( fname_list , names )

sample_number $=n p$. append ( sample_number, names.split (', , 1 ) $[0])$

else :

if names $[-9]==, 7$ '

fname $e_{-}$is t $=n p$. append ( fname $e_{-}$ist , names )

sample_number $=n p$. append ( sample_number, names.split (' , , 1 ) $[0])$

numfiles $=$ len ( fname list $\left._{-}\right)$

\#The number of files that are to be analyzed

print ('begin $\sqcup$ calculating ...')

for $i$ in range $(0$, numfiles $)$ :

data $=n p$. loadtxt ( fname_list [i ] )

\#Load the data 
\#Apply Corrections

print (' applying corrections ...')

for $\mathrm{j}$ in range $(0, \operatorname{len}(\mathrm{data}[:, 1]))$ :

$\operatorname{data}[\mathrm{j}, 1]=(\operatorname{data}[\mathrm{j}, 1]-\operatorname{thermal}[\mathrm{j}]) * \operatorname{ratio}[\mathrm{j}]$

print ('s moothing data ...')

$\mathrm{x}_{-}$factor $=0.0154$

$y_{-}$factor $=10 / \max (\operatorname{data}[:, 1])$

time $=$ data $[:, 0] * x_{\text {f }}$ factor

\#The $x$-axis of the data

func $0=$ data $[:, 1] * y_{-}$factor

\#Scale the data to be processed

\#Smooth the data first with a polynomial $\mathrm{n}_{-}$samples $=$len $($time $)$

\#Number of samples in the data transform =fft.fft (func0, $n_{-}$samples )/ n_samples

\#The Fourier transform of the function funco scaled appropriately

freq $=f f t$ fftfreq $\left(n_{-}\right.$samples $) * n_{-}$samples

\#Creates the $x$-axis for the Fourier transform $x_{-}$axis $2=n p$. arange $(\operatorname{len}($ time $))$

$x_{-}$axis $=n p . \operatorname{arange}(\operatorname{len}($ time $))-\operatorname{len}($ time $) / 2$

\#Filter frequencies with a cutoff filter

temp $=$ Triangle $($ transform, freq, 600)\#600 


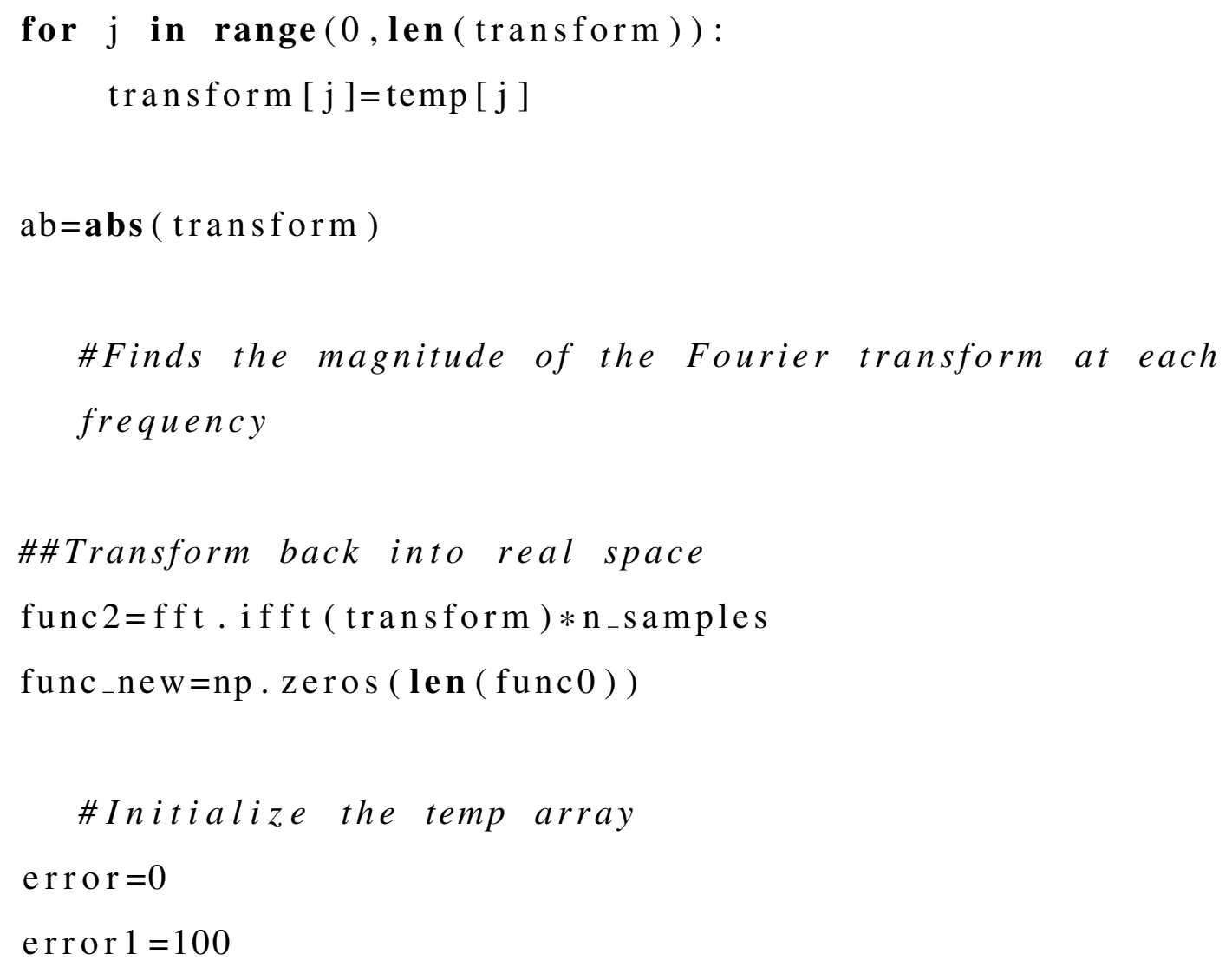


S_cen $=[1350,1640,1500,1135,1580,1880]$

Starting centers (D1, D2, D3, D4, G, G2)

Drange $=[40,40,50,90,20,30]$

\#Range of movement (D1, D2, D3, D4, G, G2) D2=35,37.5 $\mathrm{m}=\max (\mathrm{y})$

\#Find the maximum peak

$\mathrm{s}_{-} \mathrm{amp}=[4, .35,1 ., .5,2, .5]$

\#Starting amplitudes (D1, D2, D3, D4, G, L1_intercept)

for $j$ in range $\left(0\right.$, len $\left(s_{-}\right.$cen $\left.)\right)$:

$s_{-} \operatorname{cen}[j]=s_{-} c e n[j] * x_{-}$factor

\#Scaled starting centers

Drange $[\mathrm{j}]=$ Drange $[\mathrm{j}] * \mathrm{x}_{-}$factor

\#Scaled center ranges

$\left.\mathrm{s} \_a m p_{\mathrm{j}} \mathrm{j}\right]=\mathrm{s}_{-} \mathrm{amp}[\mathrm{j}] * \mathrm{~m} / 10.0$

\#Scaled starting amplitudes

\#plt.plot (data [:-5,0],y)

print (fname_list [i ] )

\#input('pause')

\#\#

plt.title (fname_list[i])

\#print ("s_cen", s_cen)

\#print ("Drange", Drange)

\#print $\left(1600 * x_{-}\right.$factor $)$ 
D1 $=\operatorname{Model}\left(\right.$ LorenztianMode1, prefix $\left.={ }^{\prime} D 1_{-}{ }^{\prime}\right)$

pars $=$ D1 . make _params $_{(}$)

\#Create parameters

pars ['D1_amp']. set (s_amp [0], $\min =0.45$ )

pars ['D1_cen' $]$. set $\left(s_{-}\right.$cen $[0], \quad \min =s_{-}$cen $[0]-$ Drange $[0], \quad \max =$

s_cen $[0]+$ Drange $[0])$

pars ['D1_Q']. set $(1000, \min =100)$

pars ['D1_F'] . set $(2, \min =.4)$

D3 $=\operatorname{Model}\left(\right.$ LorenztianModel, prefix $={ }^{\prime}$ D3 _-' $)$

pars . update (D3. make_params ())

\#Add parameters

pars ['D3_amp']. set (s_amp [2], $\min =.450)$

pars ['D3_cen' ]. set $\left(s_{-}\right.$cen [2], $\min =s_{-}$cen [2] - Drange [2], $\mathbf{m a x}=$

s_cen [2]+ Drange [2])

pars [ 'D3_Q'] . set $(1000, \quad \min =0)$

pars ['D3_F']. set $(5, \quad \min =1)$

D4 $=\operatorname{Model}\left(\right.$ LorenztianModel, prefix $={ }^{\prime}$ D4_' $)$

pars . update (D4. make_params ())

\#Add parameters

pars ['D4_amp']. set (s_amp [3], $\min =.35)$

pars ['D4_cen']. set (s_cen [3], min=s_cen[3] - Drange [3], $\max =$

s_cen [3]+ Drange [3])

pars ['D4_Q'] . set $(1000, \quad \min =10)$

pars ['D4_F'] . set $(2, \min =.5, \max =5)$ 
$\mathrm{G}=\operatorname{Model}\left(\right.$ LorenztianModel, prefix $\left.={ }^{\prime} \mathrm{G}_{-}{ }^{\prime}\right)$

pars.update (G. make_params ())

\#Add parameters

pars ['G_amp'] . set (s_amp [4], $\min =.2, \quad \max =m)$

pars ['G_cen'] set (s_cen [4], min=s_cen [4] - Drange [4]*1.1,

$\max =$ s_cen $_{-}[4]+$ Drange $[4] * 1.13$ )

pars [ 'G_Q' ]. set $(1000, \min =10)$

pars $\left[{ }^{\prime} G \_F '\right] . \operatorname{set}(.7, \min =.15, \max =1.5)$

pars . add ('Delta' $, .4, \min =0.01, \max =6) \quad \# .4$

Variable for defining relationship constraint between D2_amp and G_amp pars.add('Delta2', (s_cen[1]-s_cen $[4]) * .1, \quad \min =0.0, \max$ =2.0) \#.3 $.01 \quad$ \#Variable for relationship constraint between D2_cen and G_cen

$\mathrm{D} 2=\operatorname{Model}\left(\right.$ LorenztianModel, prefix $\left.={ }^{\prime} \mathrm{D} 2_{-}{ }^{\prime}\right)$

pars . update (D2.make_params ())

\section{\#Add parameters}

pars ['D2_amp']. set (s_amp [1], $\min =0.001, \quad \mathbf{m a x}=\mathrm{s}_{-} \mathrm{amp}$

[4]*2.15, expr='G_amp-Delta' )

pars ['D2_cen'] . set (s_cen [1], $\min =s_{-} \operatorname{cen}[1]-\operatorname{Drange}[1] * 1.2$,

$\max =$ s_cen $_{-}[1]+$ Drange $[1] * .8$, expr='Delta2 $+G_{-}$cen') $\#+.1$ pars ['D2_Q']. set $(1000, \min =10)$

pars ['D2_F' ]. set $(.75, \min =.15, \max =1.5)$ 
Line_mod=Model $($ LinearModel, prefix $=$ 'L1_' $)$

pars.update (Line_mod.make_params ())

\#Add parameters

pars ['L1_slope']. $\operatorname{set}(-0.03, \min =-.1, \max =.1)$

pars [' L 1 _intercept']. set $\left(s_{-} \_a m p[5], \quad \min =0.0, \max =5\right)$

G2 = GaussianModel(prefix ='G2_')

pars . update (G2.make_params ())

\#Add parameters

pars ['G2_center']. set (s_cen [5], $\boldsymbol{m i n}=s_{-}$cen [5]-Drange [2],

$\max =$ s_cen $_{\text {_c }}[5]+$ Drange $\left.[2]\right)$

pars ['G2_sigma' ]. set $(\min =.1, \quad \max =4)$

pars ['G2_amplitude']. set $(\boldsymbol{m i n}=0.0)$

pars [' G2_height']. set $(\min =.15)$

pars ['G2_fwhm']. set $(\min =.15, \max =10)$

$\bmod =\mathrm{D} 1+\mathrm{D} 2+\mathrm{D} 3+\mathrm{D} 4+\mathrm{G}+$ Line $\_$mod $+\mathrm{G} 2$

\#Sum the models together

\#Evaluate the models and the line fit

print ('evaluating $\_$curve ${ }_{\lrcorner}$fit $\_$model ...')

init $=\bmod . \mathbf{e v a l}(\operatorname{pars}, \quad x=x)$

out $=\bmod$. fit $(y$, pars,$\quad x=x)$

comps $=$ out.eval_components $(\mathrm{x}=\mathrm{x})$ 
print (out.fit_report (min_correl=0.5))

print (' collecting model $\sqcup$ info ...') )

$\mathrm{x}=\mathrm{x} / \mathrm{x}_{-}$factor

parameters $=[]$

parameters =np . append ( parameters , out . params.get ('L1_slope'

). value )

parameters $=n p$. append $($ parameters, out . params.get (',

L $1_{-}$intercept') . value )

parameters $=n p$. append ( parameters, out . params.get ('G2_center

'). value/ $x_{-}$factor)

parameters $=n p$. append ( parameters , out . params. get ('G2 height

'). value )

parameters $=n p$. append ( parameters , out . params . get ('D1_amp') .

value )

parameters $=n p$. append ( parameters, out . params.get ('D1_cen') . value / $x_{-}$factor )

parameters =np. append ( parameters, out . params. get ('D2_amp'). value )

parameters =np. append ( parameters, out . params. get ('D2_cen') . value / $x_{-}$factor)

parameters $=n p$. append ( parameters , out . params . get ('D3_amp') . value )

parameters $=n p$. append ( parameters , out . params . get ('D3_cen') . value / $x_{-}$factor )

parameters =np. append ( parameters, out.params.get ('D4_amp'). value )

parameters $=n p$. append ( parameters, out . params. get ('D4_cen') . value / $x_{-}$factor ) 
parameters $=n p$. append ( parameters , out . params . get ( 'G_amp') . value )

parameters $=n p$. append ( parameters , out . params.get ('G_cen') . value / $x_{-}$factor )

arguments_G2 =( out . params.get ('G2_height' ). value, out .

params.get ('G2_center'). value/ $x_{-}$factor, out.params.get ( 'G2_sigma') . value/ $x_{-}$factor )

parameters $=n p$. append ( parameters, quad (Gauss, float ('-inf'),

float (' inf'), args =arguments_G2) [0])

arguments_D1 =( out. params.get ('D1_amp') . value, out.params .

get ('D1_cen'). value/ x_factor, out.params.get ('D1_Q') .

value, out . params.get ('D1_F') . value/ $x_{-}$factor )

parameters $=n p$. append ( parameters , quad (LorenztianModel ,

float ('-inf'), float ('+inf'), args=arguments_D1 ) [0 ])

arguments_D2 $=($ out . params . get ('D2_amp') . value, out . params .

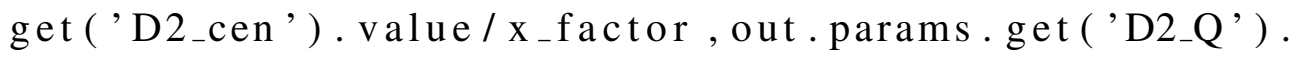

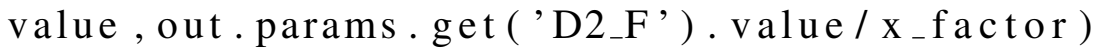

parameters $=n p$. append ( parameters , quad (LorenztianModel,

float ('-inf'), float ('+inf'), args=arguments_D2 ) [0 ] )

arguments_D3 =( out. params.get ('D3_amp') . value, out.params .

get ('D3_cen'). value/ $x_{-}$factor, out.params.get ('D3_Q').

value, out . params.get ('D3_F') . value/ x _factor)

parameters $=n p$. append ( parameters , quad (LorenztianModel,

float ('-inf'), float ('+inf'), args=arguments_D3 ) [0])

arguments_D4 =( out . params.get ('D4_amp') . value, out . params .

get ('D4_cen'). value/ X_factor, out.params.get ('D4_Q') .

value, out . params.get ('D4_F'). value/ x_factor)

parameters $=n p$. append $($ parameters , quad (LorenztianModel,

float ('-inf'), float ('+inf'), args=arguments_D4 ) [0 ]) 
arguments_G =( out . params . get ('G_amp') . value, out . params.get

('G_cen'). value/ $x_{-}$factor, out .params.get ('G_Q'). value,

out.params.get ('G_F' ). value/ $x_{-}$factor )

parameters $=n p$. append $($ parameters , quad (LorenztianModel,

float ('-inf'), float ( ' + inf'), args=arguments_G ) [0])

\#\#RECORD\#\#

print (' recording info ...')

file. write ( sample_number [i ]+", ,")

for $\mathrm{j}$ in range(len (parameters)) :

output $=\mathbf{s t r}(\operatorname{parameters}[\mathrm{j}])$

file. write ( output+", ,")

file. write ("785\n")

\#\#CHECK\#\#

$\mathrm{plot}=\mathrm{False}$

if ( out.params.get ('G_cen') . value >out . params.get ('D2_cen')

. value ):

print ("Switched")

plot $=$ True

point $=0$

for $\mathrm{j}$ in $\operatorname{range}(\operatorname{len}(\mathrm{x}))$ :

if $\operatorname{abs}\left(y[j]-o u t\right.$. best fit $\left._{[}[\mathrm{j}]\right)>.2$ :

points $=$ points +1

if points ==4:

plot $=$ True

break

if $p l o t==$ True :

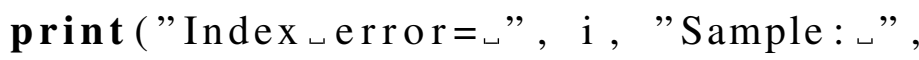

sample_number [i ])

print ( out . fit_report ( min $_{-}$correl $\left.=0.5\right)$ )

plt. figure (0) 


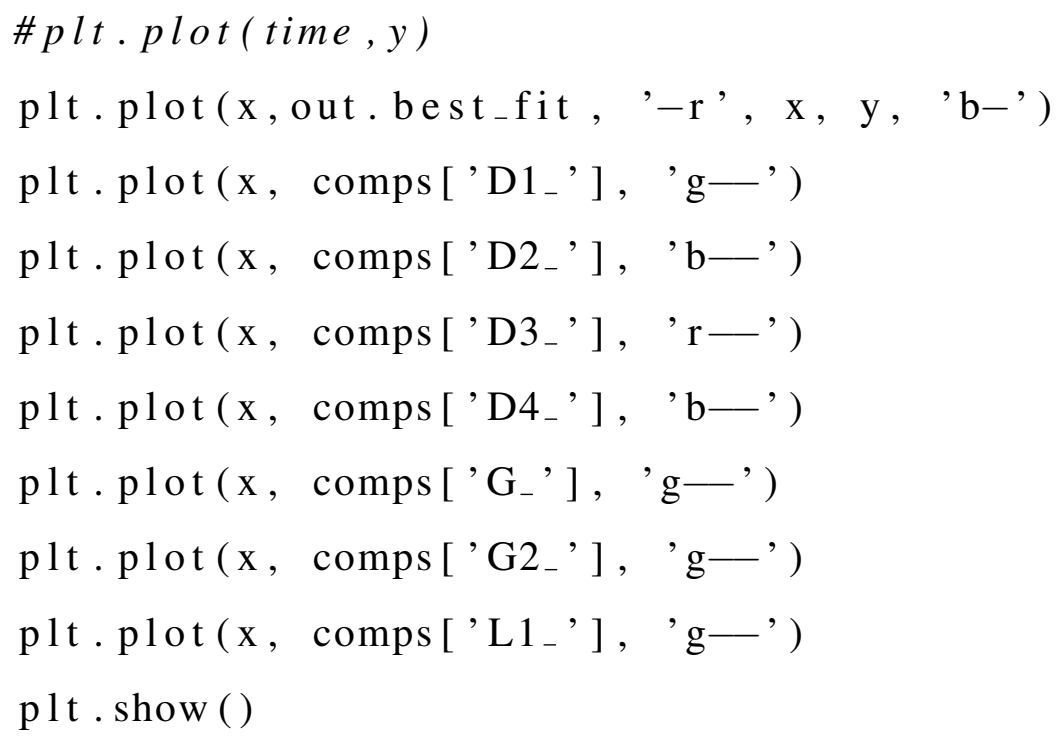

else :

print ("Index $=_{\sqcup} "$, i , " Sample: : ", sample_number [ i ] )

file. $\operatorname{close}()$

print (' done')

\#\#plt.show() 